San Jose State University

SJSU ScholarWorks

Master's Theses

Master's Theses and Graduate Research

Spring 2017

\title{
Comparative Study of Broadband Photometry Relations for Ultra- Diffuse and Normal Galaxies in the Coma Cluster
}

\author{
Maria Babakhanyan Stone
}

San Jose State University

Follow this and additional works at: https://scholarworks.sjsu.edu/etd_theses

\section{Recommended Citation}

Stone, Maria Babakhanyan, "Comparative Study of Broadband Photometry Relations for Ultra-Diffuse and Normal Galaxies in the Coma Cluster" (2017). Master's Theses. 4823.

DOI: https://doi.org/10.31979/etd.y5dm-j3p4

https://scholarworks.sjsu.edu/etd_theses/4823

This Thesis is brought to you for free and open access by the Master's Theses and Graduate Research at SJSU ScholarWorks. It has been accepted for inclusion in Master's Theses by an authorized administrator of SJSU ScholarWorks. For more information, please contact scholarworks@sjsu.edu. 
COMPARATIVE STUDY OF BROADBAND PHOTOMETRY RELATIONS FOR

ULTRA-DIFFUSE AND NORMAL GALAXIES IN THE COMA CLUSTER

\author{
A Thesis \\ Presented to \\ The Faculty of the Department of Physics and Astronomy \\ San José State University \\ In Partial Fulfillment \\ of the Requirements for the Degree \\ Master of Science
}

by

Maria Babakhanyan Stone

May 2017 
(c) 2017

Maria Babakhanyan Stone

ALL RIGHTS RESERVED 
The Designated Thesis Committee Approves the Thesis Titled

COMPARATIVE STUDY OF BROADBAND PHOTOMETRY RELATIONS FOR ULTRA-DIFFUSE AND NORMAL GALAXIES IN THE COMA CLUSTER

by

Maria Babakhanyan Stone

APPROVED FOR THE DEPARTMENT OF PHYSICS AND ASTRONOMY

SAN JOSÉ STATE UNIVERSITY

May 2017

Dr. Aaron Romanowsky Department of Physics and Astronomy

Dr. Elisabeth Mills Department of Physics and Astronomy

Dr. Thomas Madura Department of Physics and Astronomy 


\title{
ABSTRACT \\ COMPARATIVE STUDY OF BROADBAND PHOTOMETRY RELATIONS FOR ULTRA-DIFFUSE AND NORMAL GALAXIES IN THE COMA CLUSTER
}

\author{
by Maria Babakhanyan Stone
}

Ultra-diffuse galaxies are a novel type of galaxies discovered first in the Coma cluster. These objects are characterized simultaneously by large sizes and by very low counts of constituent stars. Conflicting theories have been proposed to explain how these large diffuse galaxies could have survived in the harsh environment of clusters. To date, thousands of these new galaxies have been identified in cluster environments. However, further studies are required to understand their relationship to the known giant and dwarf classes of galaxies. The purpose of this study is to compare the trends of inner and outer populations of normal members of the Coma cluster and ultra-diffuse galaxies in color-magnitude space. The present work used several astronomical catalogs to identify the member galaxies based on the coordinates of their positions and to extract available colors and magnitudes. We obtained correlations to convert colors and magnitudes from different systems into the common Sloan Digital Sky Survey system to facilitate the comparative analysis. We showed the quantitative relations describing the color-magnitude trends of galaxies in the core and the outskirts of the cluster. We confirmed that the inner and outer populations of ultra-diffuse galaxies exhibit an offset similar to the normal red sequence galaxies. We presented an initial assessment of stellar population ages and metallicities which correspond to the obtained color offsets. We surveyed the available images of the cluster for outliers, merger candidates, and candidate ultra-diffuse galaxies. We conclude that ultra-diffuse galaxies are an important part of the Coma cluster evolutionary history and future work is needed especially in obtaining spectroscopic data of a larger number of these dim galaxies. 


\section{ACKNOWLEDGMENTS}

This work is dedicated to my amazing teachers, professors, and mentors who inspire and enrich their students every day. I am extremely fortunate to have studied under dedicated, selfless, interesting, and wonderful educators. There are not enough words in the Universe to describe my gratitude for the gifts of knowledge, patience, and encouragement that these extraordinary individuals share with me as their student.

I would like to acknowledge Dr. Aaron Romanowsky, my research advisor, who gave me the opportunity to participate in cutting edge astronomy research. I am truly thankful for his leadership in challenging me to work on my own thesis project. He provided constant assistance to me as a burgeoning scientist throughout the weeks of laborious study of the astrophysics concepts and research tools to analyze and understand the photometry of ultra-diffuse galaxies. I especially appreciate the opportunity to present my research during the AAS 229 conference as a poster.

I would like to acknowledge Jin Koda and Masafumi Yagi for corresponding and answering questions regarding the catalog of UDGs. I would like to acknowledge Nobu Okabe for providing data from the Subaru telescope on the Coma cluster and for answering questions about the technical details of these observations. I would like to acknowledge also all of the astronomical research groups and organizations which made their surveys and catalogs public.

I would like to particularly thank the members of my committee for their guidance through the thesis process: Dr. Patrick Hamill, Dr. Olenka Hubickyj, Dr. Thomas Madura, and Dr. Elisabeth Mills. Their expertise and advice helped me to present my thesis work as a culminating experience of my graduate studies. I would like to thank Dr. Thomas Madura for always having his door open to answer my 
questions. I am especially grateful for the great insights, numerous suggestions, support, and encouragement during my meetings with Dr. Mills, who is a wonderful role model as a woman in astronomy.

I would like to thank the professors, staff, and students of the Department of Physics and Astronomy for providing me with a world-class education. In particular, Dr. Hamill, Dr. Wharton, and Dr. Parvin taught the core graduate courses in Physics and gave me the ability to tackle the scientific literature and textbooks for this research on my own. I would like to thank also my professors from the French program: Mme Trudeau, M Desalvo, and Mme van Hooff, for inspiring me to think outside the box especially by giving me the opportunity to study abroad in Paris, France. This work would not be possible without the diverse, supportive, and welcoming environment of the whole SJSU community.

I would also like to thank my friends and family who supported me, each in their own way, and especially my mentor Dr. Miriam Rodón for sharing with me her experience in managing a long-term science project.

I dedicate this work also to my dear and loving husband James Michael Stone. Thank you, Jamie, for your unending patience and for cheering me up constantly as I worked through my thesis.

Finally, I would like to acknowledge the bright scientists and philosophers like Archimedes, Aristotle, Newton, Kepler, Galileo Galilei, Giordano Bruno, and Copernicus, who came before me and whose work and stories sparked my interest in astronomy, so that the long hours of struggle to master the technically difficult material and research tools was actually an exhilarating journey of understanding better the enigmatic ultra-diffuse galaxies and through them the Universe. 


\section{TABLE OF CONTENTS}

\section{CHAPTER}

1 INTRODUCTION AND MOTIVATION 1

1.1 Unusual Galaxies . . . . . . . . . . . . . . . . . . . . . . 1

1.1.1 Discovery of ultra-diffuse galaxies . . . . . . . . . . . . . . . 3

1.1.2 The scope of this study . . . . . . . . . . . . 6

2 DATA SOURCES 9

2.1 Catalogs for ultra-diffuse galaxies . . . . . . . . . . . . . . . 9

2.2 Data for normal member galaxies of the Coma cluster . . . . . . . . . 10

3 TRANSFORMATION METHODS 12

3.1 Standard $B-R$ color . . . . . . . . . . . . . . . . . . 14

3.1.1 Subaru $B-R$ to the normal $B-R$ transformation . . . . . . 14

3.1.2 The SDSS ugriz to the standard $B-R$ color transformation . 15

3.2 The SDSS ugriz magnitudes . . . . . . . . . . . . . . . . . . . 16

3.2.1 The SDSS ugriz magnitudes for normal galaxies . . . . . . . . 16

3.2.2 The SDSS $g$ and $r$ magnitudes for UDGs . . . . . . . . . . . 17

3.3 Color-color transformations . . . . . . . . . . . . . 18

4 ANALYSIS AND RESULTS 19

4.1 Inconsistencies with the literature . . . . . . . . . . . . . . 19

4.1.1 Outlier UDG example . . . . . . . . . . . . . . . . . 25

4.2 Visual inspection and search for UDGs . . . . . . . . . . . . . . . 31

4.2.1 UDG candidate example . . . . . . . . . . . . . . . . . 38

4.3 Transformations pipeline . . . . . . . . . . . . . . . . . 41

4.4 Color-magnitude diagrams . . . . . . . . . . . . . . . . . 45

4.4.1 Color-magnitude diagrams of normal galaxies . . . . . . . . . 48

4.4.2 Color-magnitude diagrams of UDGs . . . . . . . . . . . . . . . 51

4.5 Stellar populations . . . . . . . . . . . . . . 56

5 SUMMARY 63

5.1 Discussion .......................... 63

$\begin{array}{ll}\text { BIBLIOGRAPHY } & 67\end{array}$ 


\section{LIST OF TABLES}

Table $4.1 \quad$ R.A. and Decl. values of candidate UDGs. . . . . . . . . . . 35 


\section{LIST OF FIGURES}

Figure 4.1 CMD plots of UDGs using different $R$ magnitudes. . . . . . . . 21

Figure 4.2 CMD plots of UDGs using Sersic1 and Sersic2 $R$ magnitudes. $\quad . \quad 22$

Figure 4.3 Corrected CMD diagram for UDGs. . . . . . . . . . . . . 23

Figure $4.4 \quad$ Outlier UDGs. . . . . . . . . . . . . . . . . . . . . . . . . . . . . 24

Figure 4.5 Yagi366 UDG in its immediate environment (SDSS image). . . . 27

Figure 4.6 Zoom into Yagi366 UDG (SDSS image). . . . . . . . . . . . 28

Figure 4.7 A further zoom into Yagi366 UDG (SDSS image). . . . . . . . . 28

Figure 4.8 No SDSS photometry is available for Yagi366 UDG. . . . . . . . 29

Figure 4.9 Yagi366 UDG and its surroundings in an image via HST . . . . 29

Figure 4.10 A further zoom into Yagi366 UDG with HST . . . . . . . . . . 30

Figure 4.11 The denser core region of Yagi366 from the HST image. . . . . 30

Figure 4.12 Image of DF 23 galaxy from the $H S T$ archive. . . . . . . . . . . 32

Figure 4.13 UDG 386, a merger candidate. . . . . . . . . . . . . . . 33

Figure 4.14 UDGs 372 and 373, a merger candidate. . . . . . . . . . . . . 34

Figure 4.15 Candidate UDG on the HSCMAP viewer with its immediate environment. . . . . . . . . . . . . . . . 39

Figure 4.16 A zoomed-in image of the candidate UDG on the HSCMAP viewer. 39

Figure 4.17 Candidate UDG in the HSCMAP2 visualization. . . . . . . . . . 40

Figure 4.18 Standard $B-R$ to the SDSS $g-i$ transformation for normal galaxies. . . . . . . . . . . . . . . . . . 42

Figure $4.19 \quad g-i$ vs. $g-r$ color-color relation . . . . . . . . . . . . . 44 
Figure 4.20 Relative positions of normal member galaxies in the Coma cluster. 45

Figure 4.21 Map of (a) all UDGs and (b) UDGs with Subaru $B-R$ data. . 47

Figure 4.22 CM trends of inner and outer populations of normal galaxies. . . 49

Figure $4.23 \quad \mathrm{CM}$ trends of inner and outer red sequences. . . . . . . . . . . . 50

Figure 4.24 CMD trend of all UDGs. . . . . . . . . . . . . . . . . . . . . 52

Figure 4.25 CMD trends for inner and outer UDGs. . . . . . . . . . . . . 53

Figure 4.26 Color-magnitude diagram of all Coma cluster galaxies. . . . . . 54

Figure 4.27 Alternative color-color relations for obtaining the SDSS $g-i$ color. 55

Figure 4.28 CMD with UDGs and normal galaxies using Okabe catalog. . . 56

Figure 4.29 Exploratory color-age space from FSPS models. . . . . . . . . . 57

Figure $4.30 \quad$ FSPS model result with one-tenth solar metallicity. . . . . . . . 58

Figure 4.31 UDGs: FSPS model result with one-tenth solar metallicity. . . . 59

Figure 4.32 Normal galaxies: FSPS model result with one-tenth solar metallicity. . . . . . . . . . . . . . . 60

Figure 4.33 Metallicities from FSPS with a 10 Gyr track. . . . . . . . . . 61

Figure 4.34 Metallicities from FSPS with a 7 Gyr track. . . . . . . . . . 62 


\section{CHAPTER 1}

\section{INTRODUCTION AND MOTIVATION}

\subsection{Unusual Galaxies}

Our current observable universe consists of numerous galaxies (two trillion galaxies at redshift $z<8$; Conselice et al. 2016). Like our own Milky Way Galaxy,

all galaxies are composed of stars and gas which are bound together through gravity (Binney \& Merrifield 1998). Early extragalactic astronomers noticed that there were different types of galaxies. The original classification of galaxies was performed by Edwin Hubble based primarily on the appearances of the galaxies; uniform symmetrical galaxies and beautiful spiral galaxies further separated into barred and non-barred categories (Hubble 1926). This classification is known as the Hubble tuning fork and it separates the elliptical galaxies and spiral galaxies into two distinct groups (Hubble 1936). The appearances of these galaxies mirror the underlying physical differences that typically exist between elliptical and spiral galaxies. The elliptical galaxies are older, redder, smoother, and featureless. The spiral galaxies are usually younger and bluer, harboring active star-forming regions. For example, our own host galaxy, the Milky Way, and its neighbor Andromeda are spiral galaxies. These giant galaxies follow the standard dichotomy of the Hubble fork.

Just as trillions of stars are grouped together through gravity to form a galaxy, the galaxies themselves form enormous entities such as groups and clusters under the influence of gravity, supporting the hierarchical model of the universe (Mo et al. 2010). For example, the Coma cluster is one of the larger clusters. It hosts numerous massive, old elliptical galaxies. Furthermore, both the Virgo cluster (distance about 16.5 Mpc; Mei et al. 2007) and the Coma cluster (roughly $100 \mathrm{Mpc}$; 
Jensen et al. 2015) are part of the Local Supercluster.

However, the universe is not limited only to the clearly defined spirals and ellipticals. Even Hubble identified irregular galaxies which did not exhibit the clear-cut features of either of the two branches. Since Hubble, other galaxies have been discovered and categorized, e.g., lenticular galaxies (van den Bergh 1979), dwarf spheroidals (Da Costa 1992), dwarf elliptical galaxies (Ferguson \& Binggeli 1994), ultracompact dwarf galaxies (Phillipps et al. 2001), compact ellipticals like M32 twins (Mieske et al. 2005), 'Green Peas' (Cardamone et al. 2009), faint metal-poor blue compact dwarfs (Griffith et al. 2011), and ultra-faint galaxies (Brown et al. 2014).

It is important to study the different types of galaxies as they are a window to the large-scale structure of the universe (Geller \& Huchra 1989); we think that galaxies trace the invisible dark matter halos (e.g., Watson et al. 2012) and most giant galaxies host black holes (Rees \& Volonteri 2007). Moreover, by discovering new galaxies, we can address the 'missing satellite' problem (Bullock 2010). Since galaxies are the building blocks of the universe, extragalactic astronomy provides us with the path to understanding the processes within and outside of galaxies towards a more complete picture of the whole universe we live in. Next, we discuss the discovery and features of the new ultra-diffuse galaxies.

The next section describes what is known currently about the ultra-diffuse galaxies and the aims of this study. Data sources are given in Chapter 2, transformations are explained in Chapter 3, the results are presented in Chapter 4, and we conclude this work with a summary in Chapter 5. 


\subsubsection{Discovery of ultra-diffuse galaxies}

It was in 2015 that a group of astronomers discovered a new type of galaxy, coined "ultra-diffuse galaxies" or UDGs (van Dokkum et al. 2015a) in the Coma cluster (hereafter DF 1 - DF 47 UDGs). The observations were performed through the Dragonfly Telephoto Array telescope. These 47 objects are extremely faint and typically old. The mean $g-i$ color is about 0.8 (van Dokkum et al. 2015a), a metric showing how different received fluxes are from different parts of the electromagnetic spectrum. These objects exhibit low surface brightness and thus contain fewer stars, yet some have the same size as the Milky Way Galaxy, e.g. one of the objects has a radius of $3.4 \mathrm{kpc}$ as measured from matched Hubble Space Telescope image data in the same paper (van Dokkum et al. 2015a). van Dokkum et al. (2015a) suggested that UDGs lost their gas early on and postulated high dark matter fractions for these objects.

The original discovery paper spurred a flurry of subsequent research to investigate further these unusual objects which conspicuously evaded the watchful eyes of the astronomers during earlier observations. This phenomenon perhaps echoed Gibson Reaves' argument (1956) that the difficulty of discovery could be a problem of recognition, not of detection, in view of the fact that even though it is challenging to observe UDGs, some earlier observations did contain UDGs amongst the other galaxies but they were not taken notice of next to their prominently bright cousins. A second paper from van Dokkum et al. (2015b) confirmed that one of the largest UDGs was a bona fide member of the Coma Cluster. UDGs are abundant in the Coma cluster: a recent paper found about 1000 UDGs in the Coma cluster using Subaru archival images (Koda et al. 2015). Koda et al. (2015) show that not all UDGs are identical and some are nucleated. The publication of this 
catalog of Coma UDGs appeared later, although it only covered a section and not the total apparent area of the Coma cluster (Yagi et al. 2016). In general, UDGs exhibit an effective radius of $r_{e}=3^{\prime \prime}-10^{\prime \prime}$ or $1.4-4.7 \mathrm{kpc}$ and a central surface brightness $\mu(g, 0)=24-26$ mag $\operatorname{arcsec}^{-2}$.

Subsequently, UDGs have been found in other clusters and even in low-density environments, i.e., outside of clusters. Thus, a tidally disrupted UDG as well as UDGs without any evidence of tidal disruption have been discovered in the Virgo cluster (Mihos et al. 2015). The observation of undisturbed UDGs lead to the question of why these diffuse objects were able to survive in a cluster of galaxies. Originally thought to be a dwarf spheroidal galaxy in an environment of lower density in a Pisces-Perseus supercluster filament, DGSAT I was confirmed to span a radial distance of about $4.7 \mathrm{kpc}$, thus falling into the ultra-diffuse category (Martínez-Delgado et al. 2016). Furthermore, a more recent spectroscopic study of five ultra-diffuse galaxies in the Coma cluster showed that one of them had emission lines and thus contained gas and sources of ionizing radiation (Kadowaki et al. 2017). Leisman et al. (2017) also reported a discovery of UDGs with HI content during the Arecibo Legacy Fast ALFA (Almost) Darks Project. They identified the stellar masses of these UDGs to be those of dwarfs, but HI amounts to match the $L^{*}$ galaxies. Other studies looked at the globular cluster populations as a proxy for mass estimations and dark matter studies, e.g., analyses by Peng et al. (2016), van Dokkum et al. (2016), and Beasley et al. (2016), the latter publication being the first of the rare studies in which the masses of UDGs were measured. The discovery of UDGs opened numerous unanswered questions about them and about their relationship to other known galaxies.

While observational astronomy groups began searching for new UDGs and re-examining the archival images inside and outside of the cluster environment, 
theorists are faced with the task of explaining the existence, formation, and evolution of these new types of galaxies. Currently these objects are considered somewhat controversial by the scientific community. For example, Amorisco and Loeb (2016) argue that they are an extreme tail of the dwarf galaxies.

There are different theories about their formation (Burkert 2017). One current school of thought is that UDGs are failed giant galaxies. Peng and Lim (2016) propose that UDGs host "pure stelar halos" as these are the stars that have been originally formed but subsequent star formation did not follow as they fell into the cluster environment. Interestingly, Janssens et al. (2017) propose a curious relationship between the UDGs and ultra-compact dwarfs during the infall of UDGs into the cluster. At the same time, Bellazzini et al. (2017) argue for an evolutionary link between dwarf irregulars and UDGs. Yet another proposed explanation is that UDGs are dwarf galaxies which were born outside of the cluster but then migrated into the cluster by gravitation (Román \& Trujillo 2017).

UDGs are also interesting objects in the field of dark matter studies as they hold higher dark matter fractions: see discussions of numerical simulations by Yozin and Bekki (2015) and more recent cosmological simulations by Di Cintio et al. (2017). In fact this theory that they are dark matter dominated is one of the explanations for why these objects survive in a cluster environment (e.g., van der Burg et al. 2016). The Extended Modified Newtonian Dynamics model-based study by Hodson and Zhao (2017) tried to explain the characteristic dark matter dominance in UDGs and suggested that more observations of UDGs are necessary. While there is not a single theory to explain the existence and ubiquity of UDGs, the fact that they are an important galaxy population is consistent amongst all of the research groups.

It is important to study ultra-diffuse galaxies because there are several explanations to why they formed and evolved into their current form. Furthermore, 
since they are so abundant, in order to understand the evolution of galaxies, we have to take into account UDGs as well. In particular, in the Coma cluster, UDGs are prevalent, even though it is an older cluster, which is usually characterized by harsh evolutionary histories of infall, disruption, and dissolution of galaxies. How the UDGs survive in the rough environment within the Coma cluster without being broken apart by mergers is a phenomenon that needs to be addressed by any theory. Lastly, since UDGs contain high fractions of dark matter, they are interesting objects for cosmological simulations and theories.

\subsubsection{The scope of this study}

However, before tackling the bigger questions, it is evident from the disparate interpretations of these objects that we need a better understanding of what these objects are in relation to other galaxies. This research work looks at the photometric properties of ultra-diffuse galaxies.

Currently published photometric studies of cluster UDGs place them in the red sequence space of the color-magnitude distribution both in the Coma cluster (Koda et al. 2015) and in several other clusters with a redshift between 0.044 and 0.063 (van der Burg et al. 2016). Thus, all of the UDGs in the Coma cluster are usually grouped into the same category in color-magnitude space. However, during our examination of the results by Koda et al. (2015) we noticed that some UDGs were located outside of the red sequence of galaxies in the color-magnitude diagram (CMD), although no explanation in that paper was given for those outlying objects.

This work utilized astronomical archives, catalogs, and databases to get the colors and magnitudes of galaxies. The recently published Yagi et al. (2016) catalog of Coma cluster UDGs provided the Subaru $B-R$ color and the Subaru $R$ magnitude measurements for a fraction of these objects. This publicly available 
dataset allowed our research group to access the necessary colors and magnitudes for the comparative broadband photometry analysis.

Previous work suggested UDG properties were related to their positions relative to the cluster center (A. Romanowsky, priv. comm.). Aaron Romanowsky separated UDGs from the Yagi catalog into inner and outer populations with respect to the cluster center: the inner galaxies were closer to the core of the cluster while the outer galaxies occupied the outskirts. He further looked at these two groups of UDGs on the color-magnitude diagram (CMD) and noticed that there was a rough offset in their CMD trends.

In this research we continued the photometric analysis of quantifying the CMD trends. We further compared them with photometric data for the normal Coma cluster galaxies obtained from the Consolandi et al. (2016) catalog and the Sloan Digital Sky Survey (SDSS) Data Release 13 (DR13) database tools (Albareti et al. 2016). We thus were able to compare how inner and outer populations of UDGs related to the inner and outer populations of other known galaxies in the Coma cluster based on the standard color-color and color-magnitude relationships. We were also able to assess whether different groups of UDGs existed in the CMD.

Our primary results included the quantitative descriptions of (1) the color offset between the inner and outer populations of UDGs, (2) the comparison between the trends of normal galaxies and UDGs, (3) a visual inspection of UDGs which did not lie on the red sequence space in the CMD diagram, (4) a search for possible UDGs in the publicly available astronomical archives, and (5) a preliminary estimation of age and metallicity intervals based on stellar population models.

Besides further improving our understanding of how the colors and magnitudes of UDGs describe these galaxies and their relation to normal galaxies, the significance of this research is that it is the precursor to further analysis of UDGs in 
current models of the galaxy formation and evolution. Since these UDGs cannot be resolved into individual stars, methodologies such as the one pioneered by the prominent extragalactic astronomer Beatrice Tinsley (1968) could be used in conjunction with our results to further explore these faint giants. A more pedagogical exposition of Tinsley's work is published later (Tinsley 1980). Furthermore, since few observations target UDGs, we contributed to the retrieval of observational data by scouting the publicly available archives to cross-match currently known UDGs and to identify potential UDGs which may be observed in the future for confirmation. 


\section{CHAPTER 2}

\section{DATA SOURCES}

The data for the galaxies were extracted from various astronomical sources. Note that the magnitude and color measurements for UDGs and normal galaxies were assessed within different photometric systems (the Subaru $B-R$ and $R$ vs. the SDSS ugriz).

\subsection{Catalogs for ultra-diffuse galaxies}

The data for UDGs came from the paper by van Dokkum et al. (2015a) and from the catalog by Yagi et al. (2016). The original discovery paper by van Dokkum et al. (2015a) outlined information on 47 UDGs in the Coma cluster. That paper also provided a set of criteria which defined UDGs. We adopted the definition of the UDG from this paper as those galaxies which had an effective radius of $r_{e}=3^{\prime \prime}-10^{\prime \prime}$ or $1.4-4.7 \mathrm{kpc}$ and a central surface brightness $\mu(\mathrm{g}, 0)=24-26 \mathrm{mag}$ $\operatorname{arcsec}^{-2}$ (adopting a distance of $97.5 \mathrm{Mpc}$ to Coma). However, the van Dokkum et al. (2015a) paper only provided right ascension (R.A.) and declination (Decl.) coordinates of a handful of UDGs as well as measurements of a single magnitude.

The subsequently released paper by Koda et al. (2015) announced the discovery of about 1000 more UDGs in the Coma cluster using archival data of Suprime-Cam (Miyazaki et al. 2002) from the Subaru telescope. However, the catalog of those objects was not released at the time of publication of the paper. Note that only a fraction of those 1000 UDGs had enough photometric data for photometry analysis. Koda et al. (2015) mapped 232 UDGs onto the $B-R$ vs. $R$ color-magnitude diagram and concluded that UDGs belonged to the red sequence of galaxies.

This catalog was released months after the publication of the Koda et al. (2015) 
paper in the Yagi et al. (2016) publication. We used the positions specified by the right ascension and declination from the Yagi catalog for UDGs. We also used the $B-R$ colors and $R$ magnitudes for 232 UDGs published in the Yagi et al. (2016) catalog but originally measured by Yamanoi et al. (2012). Only 40 out of 47 DF UDGs were covered by the Yagi et al. (2016) survey. Out of these, 11 DF UDGs had $R$-band Kron magnitude and $B-R$ color measurements based on the work done by Yamanoi et al. (2012).

\subsection{Data for normal member galaxies of the Coma cluster}

We first confirmed the membership of galaxies by using their R.A. and Decl. coordinates in J2000 published in the Consolandi catalog. On the other hand, the SDSS ugriz magnitudes were extracted from the SDSS DR13 survey database (Albareti et al. 2016).

The Coma cluster contains numerous galaxies with a large range of apparent sizes. We used the published Consolandi catalog (2016) to obtain the members of the Coma cluster. This catalog includes the giant elliptical galaxies which are usually hard to pinpoint with photometry due to the shredding phenomenon. All of our R.A. and Decl. coordinates for normal galaxies were obtained from this catalog and later cross-matched with the SDSS.

The Sloan Digital Sky Survey (York et al. 2000), hereafter the SDSS, is a very large map of the universe obtained via the 2.5 meter Sloan Foundation Telescope (Gunn et al. 2006). The data collected by the SDSS are available to the public. The SDSS also provides a multitude of astronomical research tools as well as plentiful educational materials to help students and educators learn how to use the SDSS data and services. 
This thesis research work used the most current Data Release (DR13) to date. We used the SDSS CrossID tool, which allowed a convenient way to match the corresponding coordinates with the SDSS database objects and retrieve photometric and other data on those objects. Thus, we obtained the SDSS ugriz magnitudes from this comprehensive astronomical survey. The advantage of using the SDSS ugriz magnitudes lies in the fact that it is a more commonly used measure of flux and can be readily compared to other photometric studies of galaxies.

Even though the Consolandi catalog provided $g$ and $i$ magnitudes, we used the derived SDSS ugriz magnitudes for normal galaxies, because we needed at least $g, i$, and $r$ magnitudes for the transformations as described in the next chapter. Moreover, this provided to us a more consistent set of magnitudes. 


\section{CHAPTER 3}

\section{TRANSFORMATION METHODS}

In order to compare normal galaxies and UDGs in color-magnitude and color-color space, the magnitude measurements should be converted to the same system. The aim of this section is to derive the necessary colors and magnitudes to construct the SDSS $g-i$ vs. the SDSS $r$ color-magnitude plot for ultra-diffuse and normal galaxies in the Coma cluster.

To study galaxies in this research, we used previously published broadband photometry. Photometric data show how much light is received from an object in certain intervals of the electromagnetic wavelength continuum. The light is collected using different telescopes and instruments which use specialized passbands to filter through only specific bands of the spectrum. Astronomical magnitudes here correspond to specific filters and express these measurements of collected light. Astronomical colors are defined as differences in magnitudes.

The conventional system of magnitudes is the $U B V R_{C} I_{C}$ Johnson / Kron-Cousins system (Bessell 2005; Landolt 1983). It is a combination of the Johnson $U B V$ magnitude system (Johnson \& Morgan 1953) and the $R_{C} I_{C}$ Kron-Cousins system (Cousins 1976). The $U$ band is the bluest and has the shortest peak wavelength. The $I_{C}$ is in the infrared and has the longest peak wavelength.

However, each telescope (or instrument) typically has its own set of filters resulting in instrumental magnitudes. Thus, photometric transformations are carried out in order to convert the instrumental magnitudes to the standard system of magnitudes.

$B-R$ colors and $R$ magnitudes of UDGs as given by the Yagi et al. (2016) catalog came from the measurements performed by Yamanoi et al. (2012) from the 
Suprime-Cam (Miyazaki et al. 2002) of the Subaru Telescope (Iye et al. 2004). Therefore, since the measurements for UDGs were performed via a different telescope-instrument-filter system, it was necessary to convert the non-standard Subaru instrumental magnitudes to a standard system of magnitudes.

Furthermore, a second set of transformations is needed to then obtain the SDSS magnitudes. We employed the SDSS ugriz system of magnitudes as the common system. Here, the bands range from ultraviolet $(u)$ to optical $(g, r)$ to infrared $(i$, $z)$. This system is widely used by the scientific community and is readily comparable to other research on galaxies. Moreover, the SDSS survey used the SDSS ugriz system to survey a significant portion of the sky. Transformations exist between the conventional Johnson / Kron-Cousins magnitudes and the SDSS magnitudes (Jester et al. 2005). We used several published transformation equations, a linear fit statistical technique, and algebraic derivation techniques to obtain the SDSS $g-i$ color and the SDSS $g$ and $r$ magnitudes for UDGs, as an important step before the comparative analysis of the photometric trends between UDGs and normal galaxies in the Coma cluster.

Besides differences in filters, there are also two systems for zero-point calibration. The Vega system considers the star Vega's magnitudes to be zero. Since Vega outputs different fluxes in different passbands, the zero points between the filters are different. The AB system defines a flux of 3,631 Jy to be the zero point for any frequency.

The Koda et al. (2015) data are on the AB system. The SDSS filters are also on the AB magnitude system. The Suprime-Cam $B$ and $R$ filters as well as the Johnson / Kron-Cousins $U B V R_{C} I_{C}$ filters are on the Vega system. 


\subsection{Standard $B-R$ color}

To convert to the desired SDSS ugriz magnitudes, we first obtained the Subaru $B-R$ vs. the normal $B-R$ color-color relation. Then, we obtained the $B-R$ vs. the SDSS $g-i$ relation using the data of normal galaxies. We combined these two relations to get the $g-i$ colors for UDGs published in the Yagi et al. (2016) catalog from the Subaru $B-R$ colors. Recall that only 232 UDGs in the Coma cluster had color and magnitude measurements available.

\subsubsection{Subaru $B-R$ to the normal $B-R$ transformation}

The following steps outline the procedure we used to derive the approximate offset relationship between the Subaru $B-R$ color and the standard $B-R$ color. This procedure allowed then a comparison between the UDGs and the normal galaxies.

We obtained fits to the red sequence in two different filter sets: standard and Subaru. The equations were set to be equal in order to transform between the filter sets. The goal was to obtain a relationship between the Subaru $B-R$ and the standard $B-R$ colors, so that we could use the Subaru $B-R$ colors for UDGs in our analysis.

The first fit to the red sequence was in the Subaru instrument filters. The Subaru $B-R$ relation to the $R$-magnitude per Yamanoi et al. (2012) is expressed in the following equation:

$$
(B-R)_{\text {Subaru }}=(-0.029 \pm 0.010) R+(1.55 \pm 0.21)
$$

The second fit to the red sequence was done in the standard photometric system. Adami et al. (2006) provided a similar relation for the normal $B$ and $R$ 
magnitudes as described in the equation below:

$$
(B-R)_{\text {normal }}=(-0.045 \pm 0.028) R+(2.27 \pm 0.48)
$$

By setting $R=0$ in both equations, the difference between the Subaru and the normal $B-R$ colors was derived.

$$
(B-R)_{\text {normal }}=(B-R)_{\text {Subaru }}+0.72 \pm 0.52
$$

The uncertainty term in equation 3.3 was calculated using the error propagation rule of adding the uncertainties in equation 3.1 and equation 3.2 in quadrature. The significance of equation 3.3 was that we could use it to convert the eclectic Subaru $B-R$ color measurements of the UDGs to the standard $B-R$ colors.

\subsubsection{The SDSS ugriz to the standard $B-R$ color transformation}

To convert between the $B-R$ and $g-i$ colors the Jester et al. (2005) transformation equations were used. Even though these transformation equations were for stars, we employed them for the color-magnitude conversions of galaxies.

From Jester et al. (2005) we adopted the relations for $B, B-V$, and $V-R$ for all stars with $R c-I c<1.15$ which are reproduced below:

$$
\begin{aligned}
& B=g+0.39(g-r)+0.21 \\
& B-V=0.98(g-r)+0.22 \\
& V-R=1.09(r-i)+0.22
\end{aligned}
$$


We obtained $B-R$ by summing $B-V$ and $V-R$ as follows:

$$
B-R=0.98 g+0.11 r-1.09 i+0.44
$$

Thus, equation 3.7 gives us the $B-R$ color transformation from the SDSS gri magnitudes. (We used these equations for normal galaxies of the Coma cluster.) It was important to be able to transform $B-R$ to the SDSS system because then we were able to compare the photometric measurements between different systems. We used this relationship further on in the analysis to correlate the SDSS $g-i$ color and the standard $B-R$ color for normal galaxies in the Coma cluster. The next section provides methods to obtain the different magnitudes used in the color transformation equations.

\subsection{The SDSS ugriz magnitudes}

In this section, we quickly review the cross-matching parameters of the CrossID

tool to obtain the SDSS ugriz magnitudes of normal galaxies based on the positions of member galaxies identified by Consolandi et al. (2016). Then, we review the short derivation for the $g$ and $r$ magnitudes for UDGs based on the offset relationship between the SDSS $g$ and the Subaru $R$ given by Koda et al. (2015), although it is a rough and not an exact relationship.

\subsubsection{The SDSS ugriz magnitudes for normal galaxies}

Equation 3.7 called for three SDSS magnitudes: $g, r$, and $i$. However, the Consolandi et al. (2016) catalog of galaxies (containing 5771 objects) only provided two of three: $g$ and $i$. The complete set of the SDSS ugriz magnitudes was obtained via the CrossID tool in the SDSS DR13 (Albareti et al. 2016). 
To cross-match and identify the galaxies via the CrossID tool, the R.A. and Decl. coordinates from the Consolandi et al. (2016) catalog were used. The search radius setting of the CrossID matching was 1.3 arcsec. The SDSS supplied several sets of magnitudes: we selected the Model Magnitudes (modelMags) which provided the optimum colors for extended objects in all bands.

The resultant table of matched objects with the desired SDSS magnitudes contained all but 330 (out of 5771) Consolandi galaxies. We manually checked three of these non-matched galaxies in the SDSS database. The identification numbers were: SDSSJ104250.9+254415, SDSSJ104852.1+265655, and SDSSJ105022.7+264406. These did not show in the SDSS database. The probable explanation could be that some galaxies do not have the same coordinates between the DR7 version of the SDSS used by Consolandi et al. (2016) paper and the DR13 version of the survey used in this research. Furthermore, the SDSS photometry sometimes misses objects in the sky, which could contribute to the number of non-matches. Lastly, the Consolandi et al. (2016) catalog contained both Coma and Virgo cluster members. Only the galaxies in the Coma cluster were selected for further analysis in this study.

\subsubsection{The SDSS $g$ and $r$ magnitudes for UDGs}

We adopted the rough transformation provided by Koda et al. (2015) between the SDSS $g$ and the Subaru $R$ magnitudes for the red sequence of galaxies in the Coma cluster:

$$
g-R \sim 0.8
$$

However, we assumed in this case an exact equality. Isolating the unknown $g$ 
magnitude on the left hand-side, we obtained the following equation:

$$
g=R+0.8
$$

Subsequently, the $r$ magnitude was derived using the following relationship,

$$
r=g-(g-r)
$$

Equation 3.10 is a transformation which provides the values for the horizontal axis (luminosity measure) of the color-magnitude diagram for the ultra-diffuse galaxies. However, to obtain the remaining $g-i$ and $g-r$ colors for UDGs we used the data for normal galaxies and linear fit models as outlined in the next section of the methods.

\subsection{Color-color transformations}

Here we just note that to obtain the remaining pieces of the color-magnitude diagram puzzle, we used the colors and magnitudes of normal galaxies to obtain algebraic linear fit models for these correlations. The statistical package used in this research work was the scipy.stats.linregress function which carried out a standard linear least-squares regression calculation (SciPy open-source software). Linear fit statistical models allowed us to obtain $B-R$ vs. $g-i$ and $g-i$ vs. $g-r$ color-color relationships. 


\section{CHAPTER 4}

\section{ANALYSIS AND RESULTS}

\subsection{Inconsistencies with the literature}

In this section, we discuss the reproduction of a general color-magnitude diagram for UDGs based on the Yagi et al. (2016) catalog data. The latter catalog is important because it is one of the few catalogs which contains colors and magnitudes for UDGs. However, our re-created color-magnitude plot did not agree with the literature. Through private correspondence with the authors of the Yagi et al. (2016) paper, we were able to obtain helpful corrections of the catalog which allowed us to match closely the color-magnitude relations of UDGs as was published before in the Koda et al. (2015) paper.

Another reason to re-create this plot was the fact that upon closer examination of the UDGs in the color-magnitude diagram (CMD) presented by Koda et al. (2015), we noticed that some UDGs were in the blue cloud, yet the authors did not explain why these were outside of the red sequence. One of the possible explanations could be that these were misidentified UDGs. Another possible explanation is that UDGs might not all be "red and dead". It is evident that more investigation is needed to understand where UDGs are on the color-magnitude diagram in comparison with other galaxies in the Coma cluster. The outlier "bluer" UDGs were visually inspected in this research.

As the first step to investigate the UDGs, the color-magnitude plot of the Koda et al. (2015) paper was re-created using the data from the Yagi et al. (2016) catalog. It is shown in Figure 4.1a. The black line in this plot shows the red-sequence fit to the Coma galaxies (Yamanoi et al. 2012). The width of the red sequence was also identified by the $\Delta(B-R)= \pm 0.2$ upper and lower boundaries (Yamanoi et al. 
2012) and is represented by the broken lines in Figure 4.1a.

Our results did not show the same exact distribution of UDGs in the $B-R$ vs. $R$ color-magnitude space as Koda et al. (2015). It was possible that this discrepancy was due to some changes in the data between the publication of Koda's paper and the subsequent publication of the Yagi paper with the catalog. The three other diagrams (Figures 4.1b, 4.2a, and 4.2b) show the same plot but with SExtractor, Sersic1, and Sersic2 $R$ magnitudes obtained from the Yagi catalog. These plots came closer to the original CMD plot in the Koda et al. (2015) paper, but none was an exact match. 


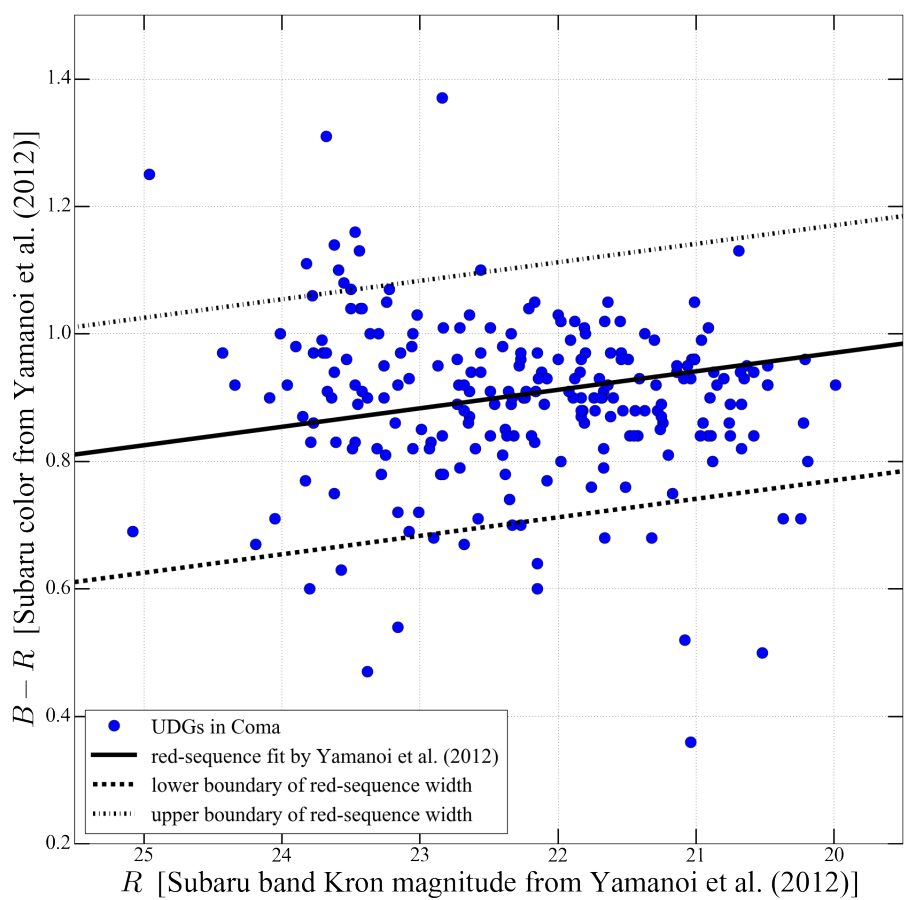

(a)

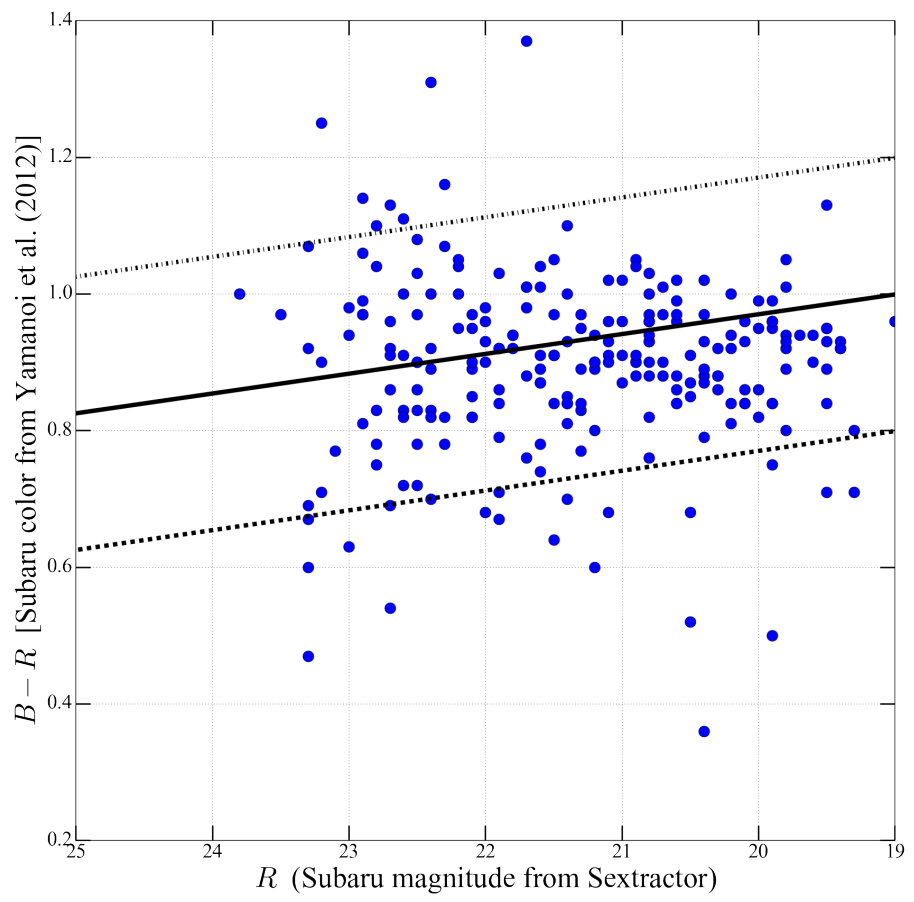

(b)

Figure 4.1: CMD plots of UDGs using different $R$ magnitudes. 


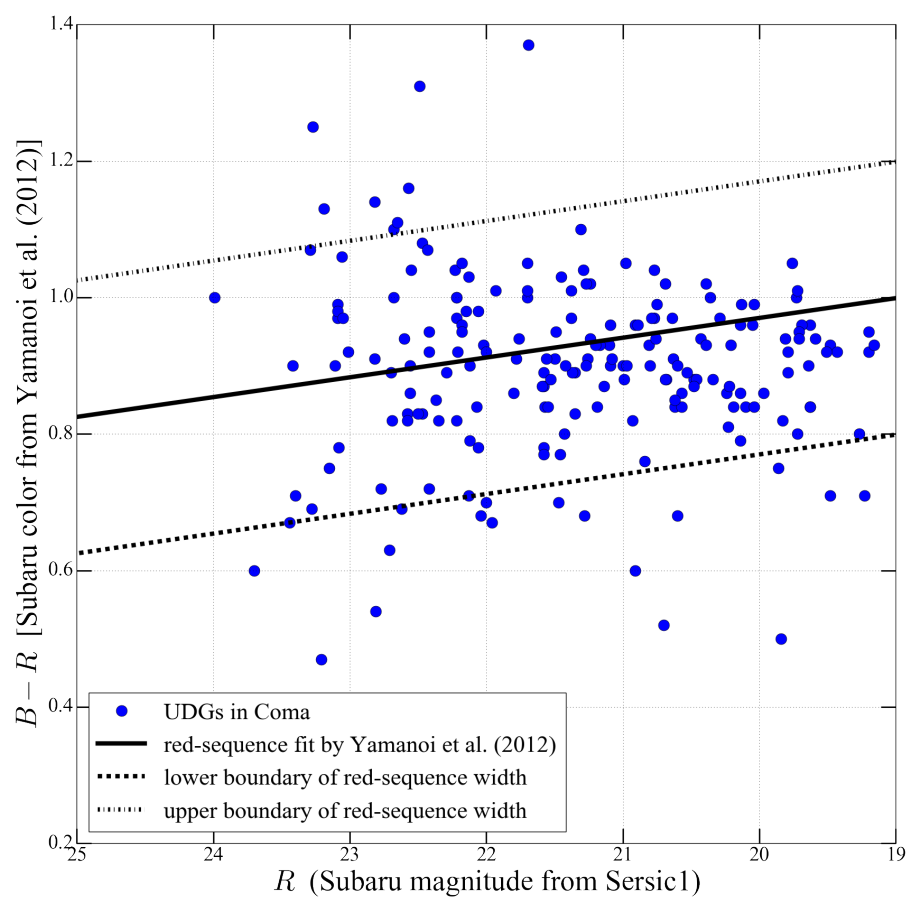

(a)

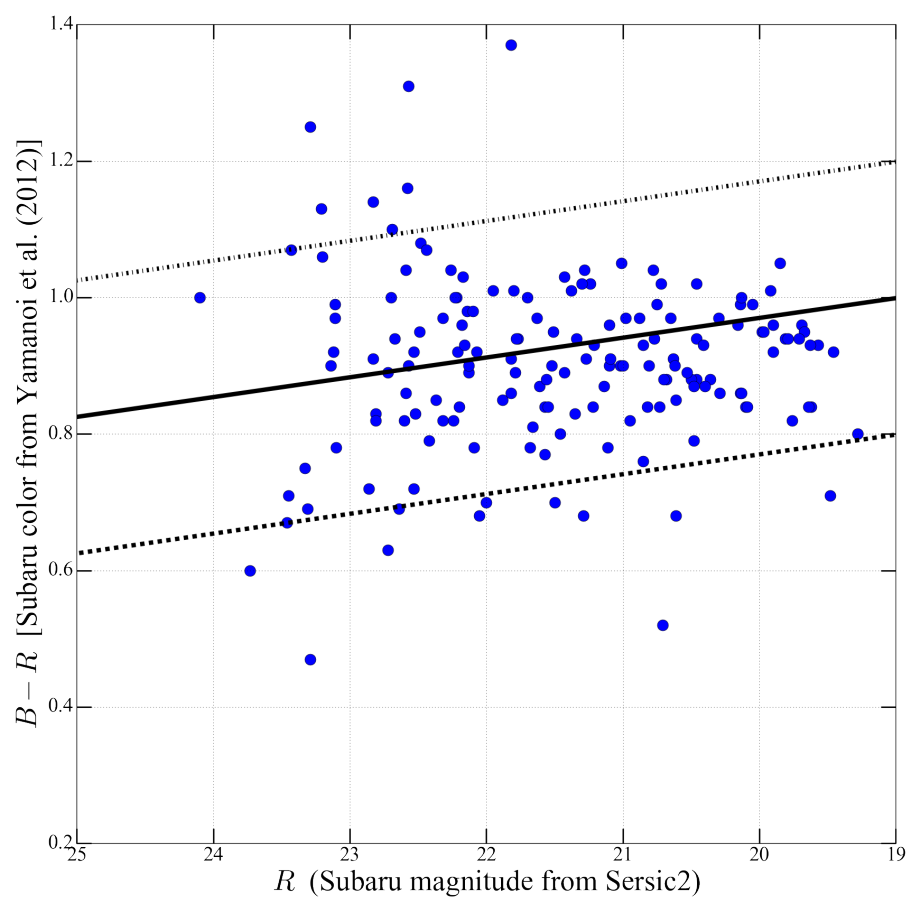

(b)

Figure 4.2: CMD plots of UDGs using Sersic1 and Sersic2 $R$ magnitudes. 
Yagi's research group shared with us that the published catalog had a mislabeled table column and that the $R$ magnitude values were actually $B$ magnitudes (priv. comm.). We adopted this change in our subsequent work. However, there were still minuscule differences between our re-worked plot with the new magnitudes and the originally published plot, and we continue to discuss this issue with the authors of the catalog.

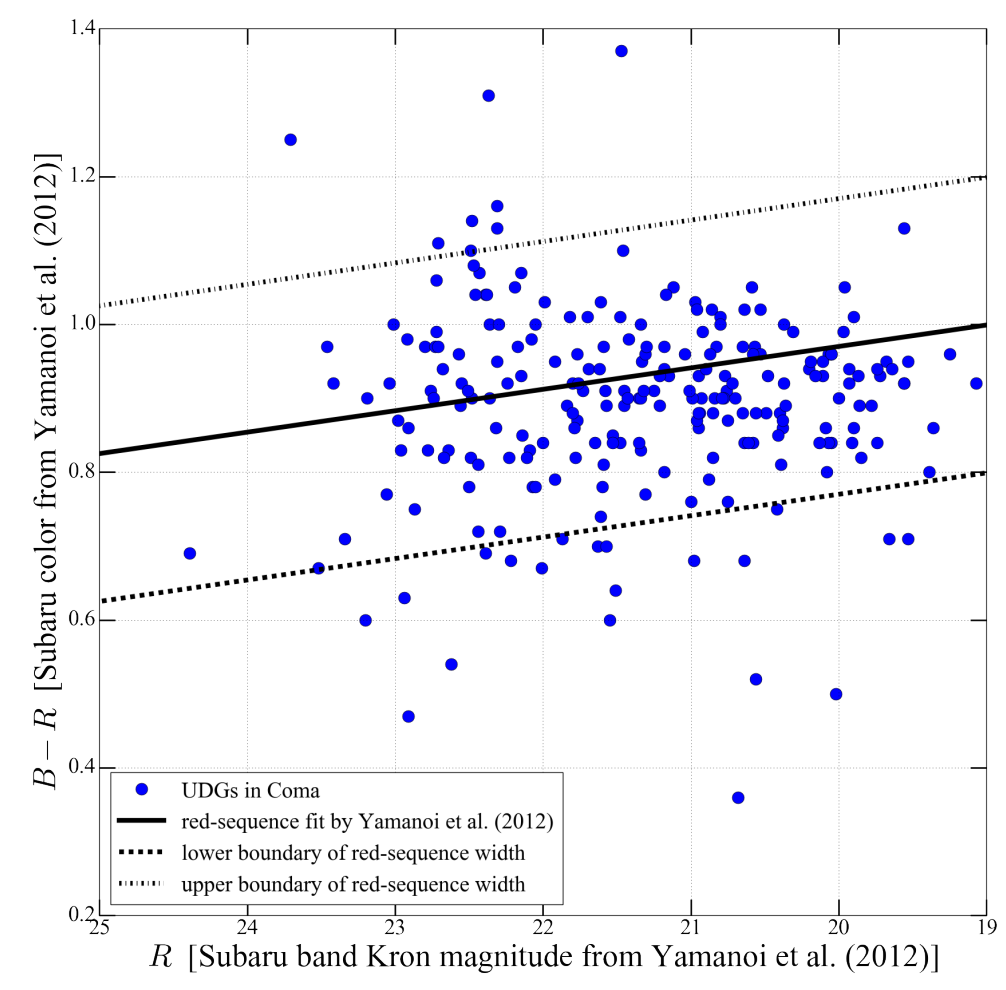

Figure 4.3: Corrected CMD diagram for UDGs.

Figure 4.3 allowed us to identify all of the outlier UDGs for which the photometric data were available. These UDGs are considered outliers because they are not within the red sequence boundaries. They lie below the lower boundary of the red-sequence band and therefore have bluer colors. Outlier UDGs are presented as red squares in Figure 4.4. The Yagi et al. (2016) catalog numbers of outlier 
UDGs are: 104, 107, 109, 252, 328, 365, 377, 424, 499, 506, 512, 513, 518, 572, 580, $582,584,670,773,783$. There are 20 outliers.

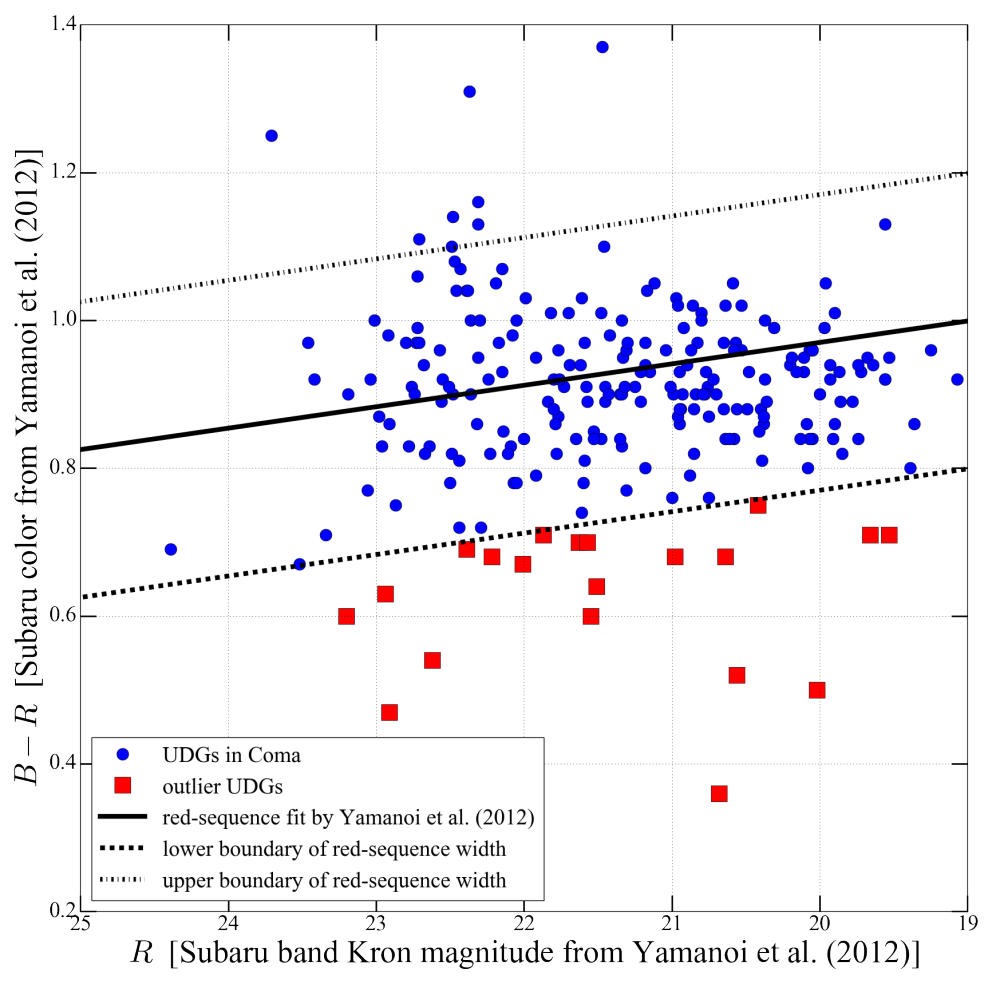

Figure 4.4: Outlier UDGs.

We conducted an eyeball survey of these outlier UDGs using several images from Nobu Okabe (priv. comm. 2017). We created a regions file in which each outlier's center was surrounded by a circle with 5 arcsec radius. The regions file was overlaid onto Okabe's FITS images of Coma10, Coma12, Coma22, and Coma41 fields (2014) via the DS9 image processing tool. The DS9 is a software tool which allows cross-matching a FITS image with a set of astronomical coordinates using a regions file.

Coma22 field contained 9 outliers: 110, 108, 105, 425, 378, 366, 329, 671, and 253. Coma41 field contained 2 outliers: 784, 774. Coma12 field had 4 outliers: 110, 
108, and 105, which overlapped with Coma22 field. Coma10 did not have any of the outliers. Therefore, out of 20 outliers, we had images of 11 to manually inspect by eye. All of these were eyeballed. All 20 outliers were searched using the SDSS DR13 Navigate tool. The outliers were faint. Several outliers had other objects within the 5 arcsec radius of their circle. Those objects could be foreground stars, for example.

Outlier 105 had an elongated tail-like protrusion. This protrusion might be an indicator of a merger. It is possible that a merger initiated star formation which would result in a bluer color for this galaxy. Outlier 425 had a bright object nearby. No spiral arms were observed in these 11 outliers. It does appear that perhaps some of these could be dwarfs, matching a few galaxies cataloged also in the Adami et al. (2006) survey, but further confirmation is needed. The outlier with the largest blue color was 366 .

Outliers 500 and 507 (DF 40) were matched with the HHM2010 GALEX survey. Outlier 514 looked blue in the SDSS and had two "more concentrated" areas. Outlier 591 also appeared blue in the SDSS and had a red star neighbor. Outlier 573 was distinctly blue in the SDSS and was also matched with the HHM2010 GALEX survey. Outlier 581 was the DF 39 object. Outlier 583 appeared to be a very extended object in the SDSS. Outlier 585 was also very extended and was matched with the HHM2010 GALEX survey. Furthermore, outlier 585 had a close galaxy nearby, and in the SDSS a protrusion appeared to connect these two galaxies. The SDSS did not detect a few of these outliers.

\subsubsection{Outlier UDG example}

We discuss one of the outliers here as an example (Yagi366 UDG). It is the "bluest" UDG on the $g-i$ vs. $r$ CMD with color-magnitude coordinates of $(g-i=0.63065, r=21.41223)$. Its R.A. and Decl. position coordinates are 194.8623 
and 27.8601 degrees. Since it is an inner UDG, it is interesting to see why it is so "blue" compared to the other galaxies, as usually the galaxies closer to the core of the Coma cluster are redder. The Yagi et al. (2016) catalog cross-matches Yagi366 UDG with several other catalogs with identifying object names:

IBG-125926+275136, APS HdBVK, YKK1-62140.

We obtained pictures of how this UDG looked through the SDSS. Figure 4.5 shows the immediate environment of this UDG in the Coma cluster. Its light is virtually invisible at that zoom. Figures 4.6 and 4.7 show a zoomed in view of the galaxy. We can distinguish the blue tints in this image. This is somewhat unusual for the UDGs since they are considered to be red. Therefore, further investigation is necessary to check whether it is truly a UDG or a blue spiral galaxy, or perhaps all UDGs are not "red and dead". Figure 4.8 shows that neither photometric nor spectroscopic data are available for this object from the SDSS, thus showing that these objects are very faint and elude detection.

Furthermore, there is another brighter galaxy in proximity to the Yagi366 UDG. It is possible that if this brighter galaxy is part of the Coma cluster that it is interacting/disrupting this UDG and maybe even through this disruption triggering star formation events. However, through our calculation of the Coma cluster redshift range, it is in the interval $0.019038<z<0.028176$. On the other hand, the NED redshift $(z=0.016700)$ for the brighter neighbor galaxy is just below the low-end $z$-value for the Coma cluster members. This indicates that there is a lower likelihood that these two galaxies interact if Yagi366 is a Coma member UDG and the brighter neighbor is outside of the Coma cluster. Hence, an alternative explanation is needed for the "blueness" of this inner UDG in the Coma cluster. If it turns out to be a miscategorized UDG, we need to re-examine the UDG catalog to make sure we are not over/under-estimating the population size of these novel galaxies. 
An additional quick look through the Hubble Space Telescope (HST) archive reveals a very blue faint elongated band/ "smudge" without an apparent shape (Figure 4.9, Figure 4.10). It appears to be a bit denser than a typical extremely faint UDG based on our observational experience, but confirmation is needed beyond our visual assessment (Figure 4.11). (In fact, there is another galaxy close by whose luminosity/stellar density looks more like that of a UDG). It appears to have a denser core region as well as at least one potential globular cluster-like region in its immediate vicinity. A more thorough confirmation is needed. It is not readily obvious via the HST image whether or not there is an interaction between those two galaxies.

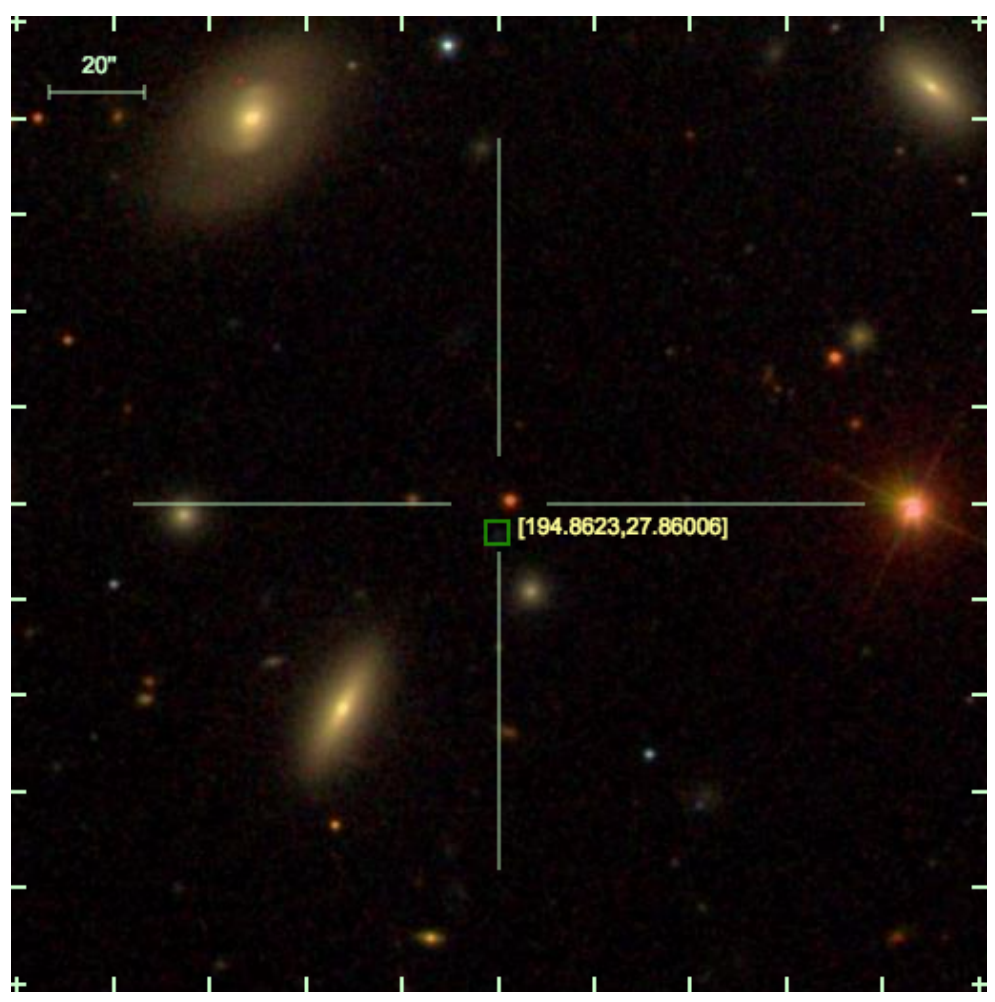

Figure 4.5: Yagi366 UDG in its immediate environment (SDSS image). 


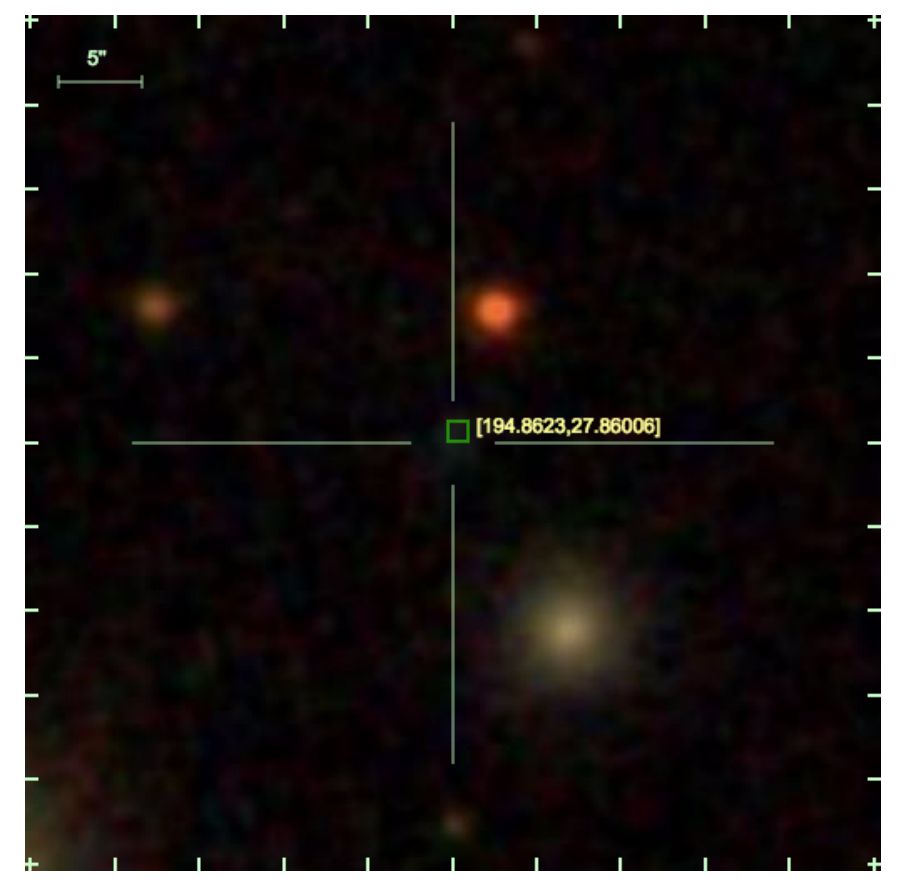

Figure 4.6: Zoom into Yagi366 UDG (SDSS image).

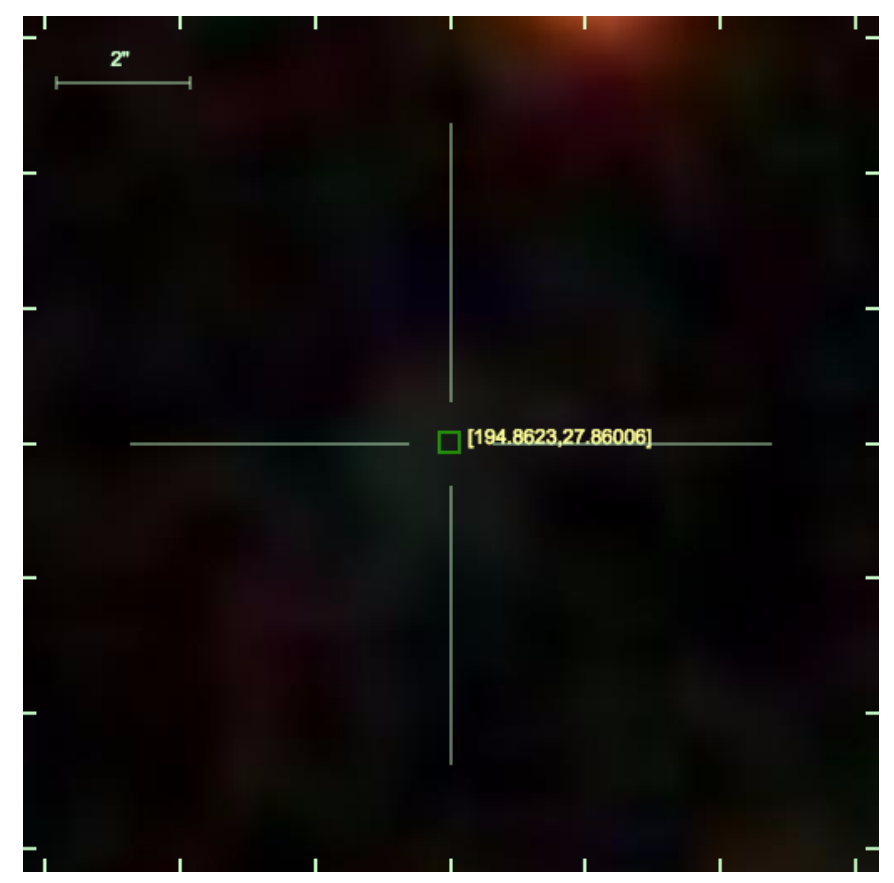

Figure 4.7: A further zoom into Yagi366 UDG (SDSS image). 


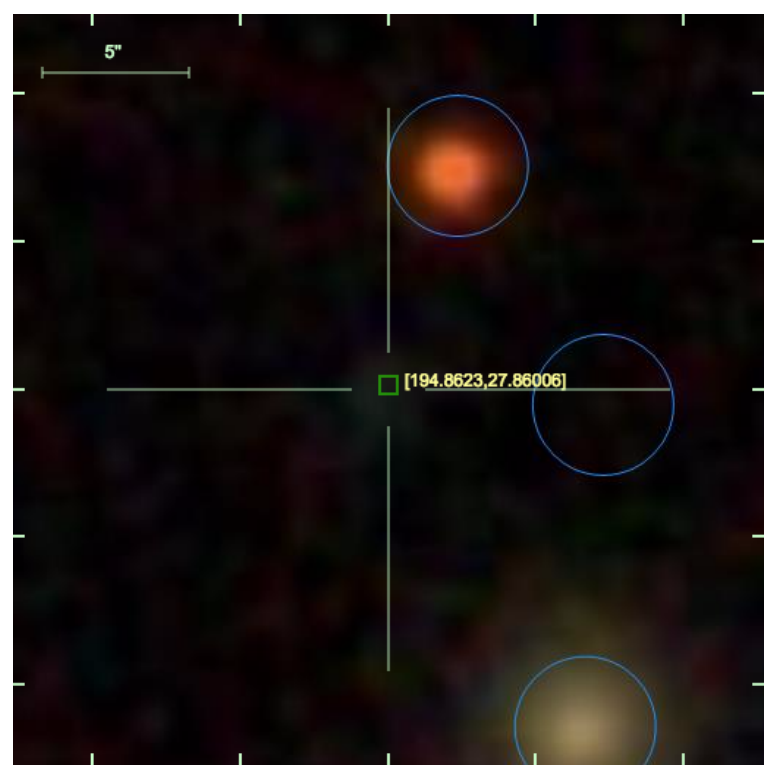

Figure 4.8: No SDSS photometry is available for Yagi366 UDG.

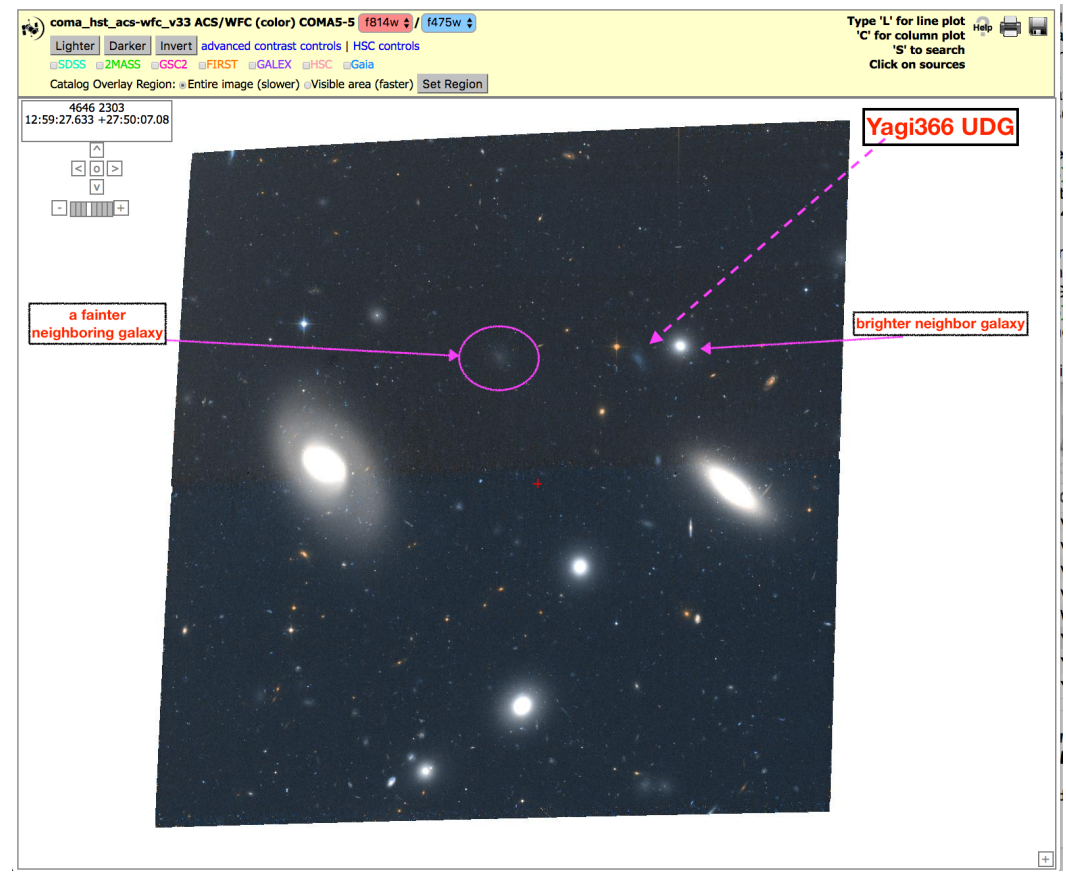

Figure 4.9: Yagi366 UDG and its surroundings in an image via $H S T$. 


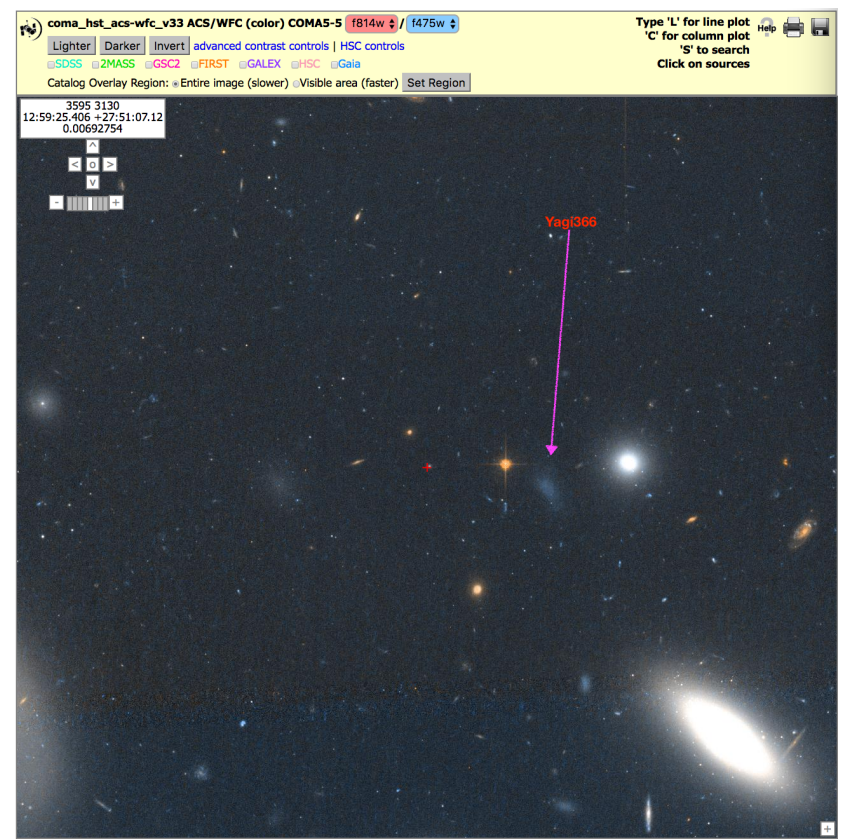

Figure 4.10: A further zoom into Yagi366 UDG with $H S T$.

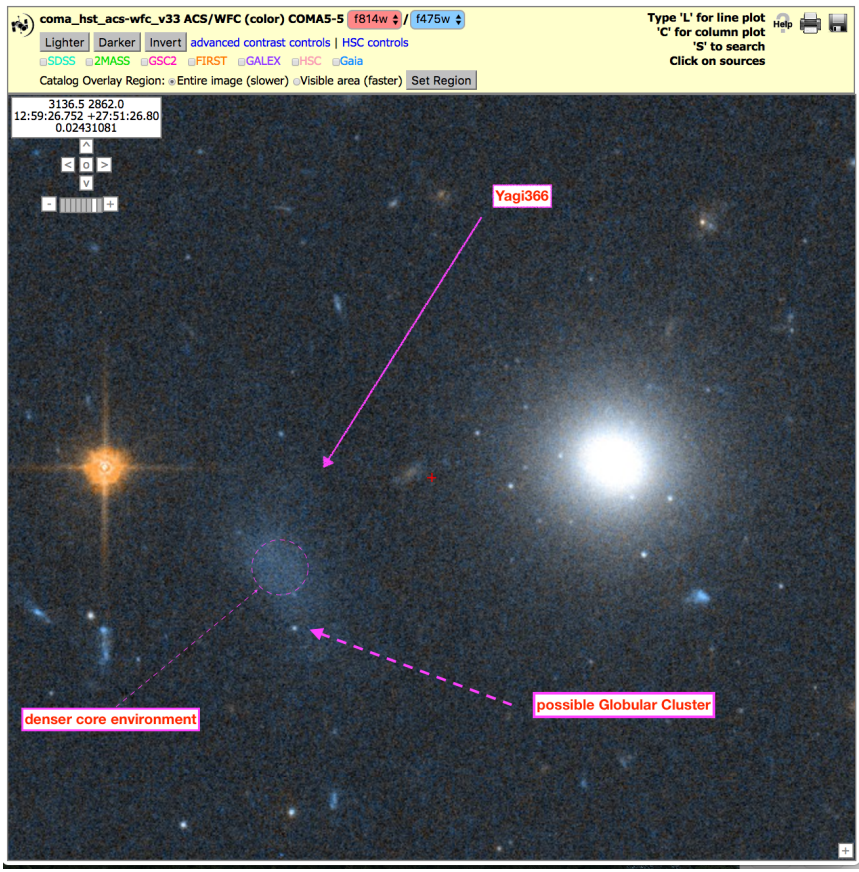

Figure 4.11: The denser core region of Yagi366 from the HST image. 


\subsection{Visual inspection and search for UDGs}

We started a preliminary visual inspection of UDGs. First, we looked at the original 47 DF UDGs from the van Dokkum et al. (2015a) publication. Then, we expanded our search to UDGs from the Yagi et al. (2016) catalog in the Coma cluster. Next, we searched for more UDGs in the Coma cluster and beyond.

The SDSS images show that DF UDGs are often too "spread out" for the SDSS to recognize them as one single photometric object. This limitation was first noticed by Dr. Romanowsky, and the search in this work also was challenged by this observational obstacle. After inspecting DF UDGs through the SDSS, we searched for those UDGs in the HST Legacy Archive based on their coordinates. The van Dokkum et al. (2015a) publication stated that only DF 17 had a match in the $H S T$ archive. However, we found that another DF UDG from van Dokkum et al. (2015a) had an image in the HST archive, DF 23 (Figure 4.12). We had to invert the color scheme and make the image significantly darker before being able to observe by eye this UDG. 
fits2web image viewer (05997_a1 WFPC2 F606W (combined) GAL-125930+274729)

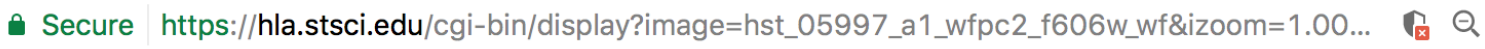

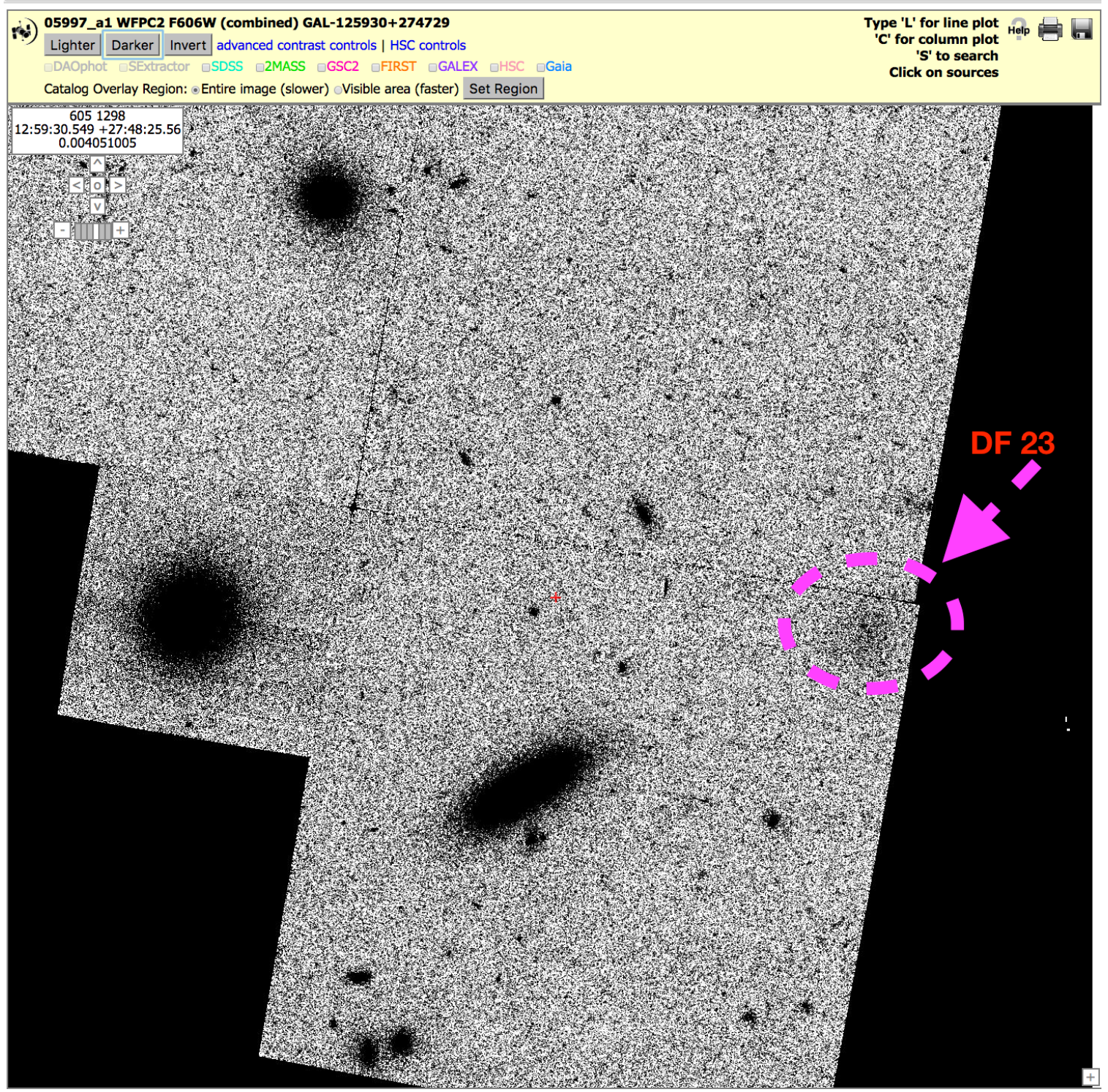

Figure 4.12: Image of DF 23 galaxy from the $H S T$ archive.

Since Koda et al. (2015) only covered a fraction of the Coma cluster area, we hoped to find more UDGs in the section of the Coma cluster missed by that publication. Visual inspection of the Yagi et al. (2016) catalog UDGs revealed two 
merger candidates. UDG 386 had an extended bipartite shape and therefore raised a question of whether or not it was a merger of two galaxies, e.g., a dwarf and a UDG, or two UDGs. See Figure 4.13, with UDG 387 nearby.

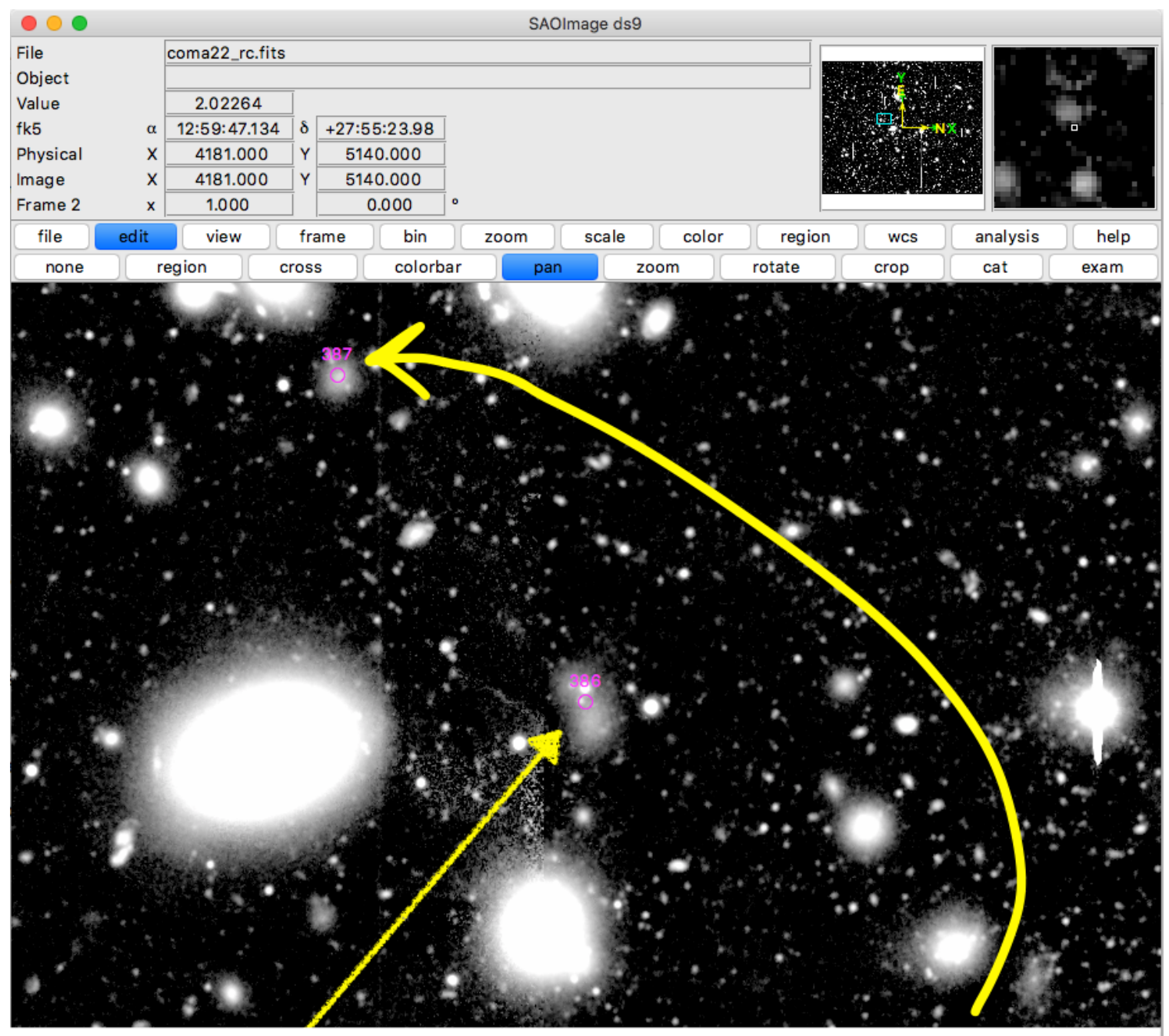

UDG 386 appears to be bilobial, compare with UDG 387 nearby.

Figure 4.13: UDG 386, a merger candidate.

UDGs 372 and 373 were observed to be in proximity to each other. The 
apparent short distance between them indicates that they might be interacting UDGs. The pair is presented in Figure 4.14 below (image from Nobu Okabe).

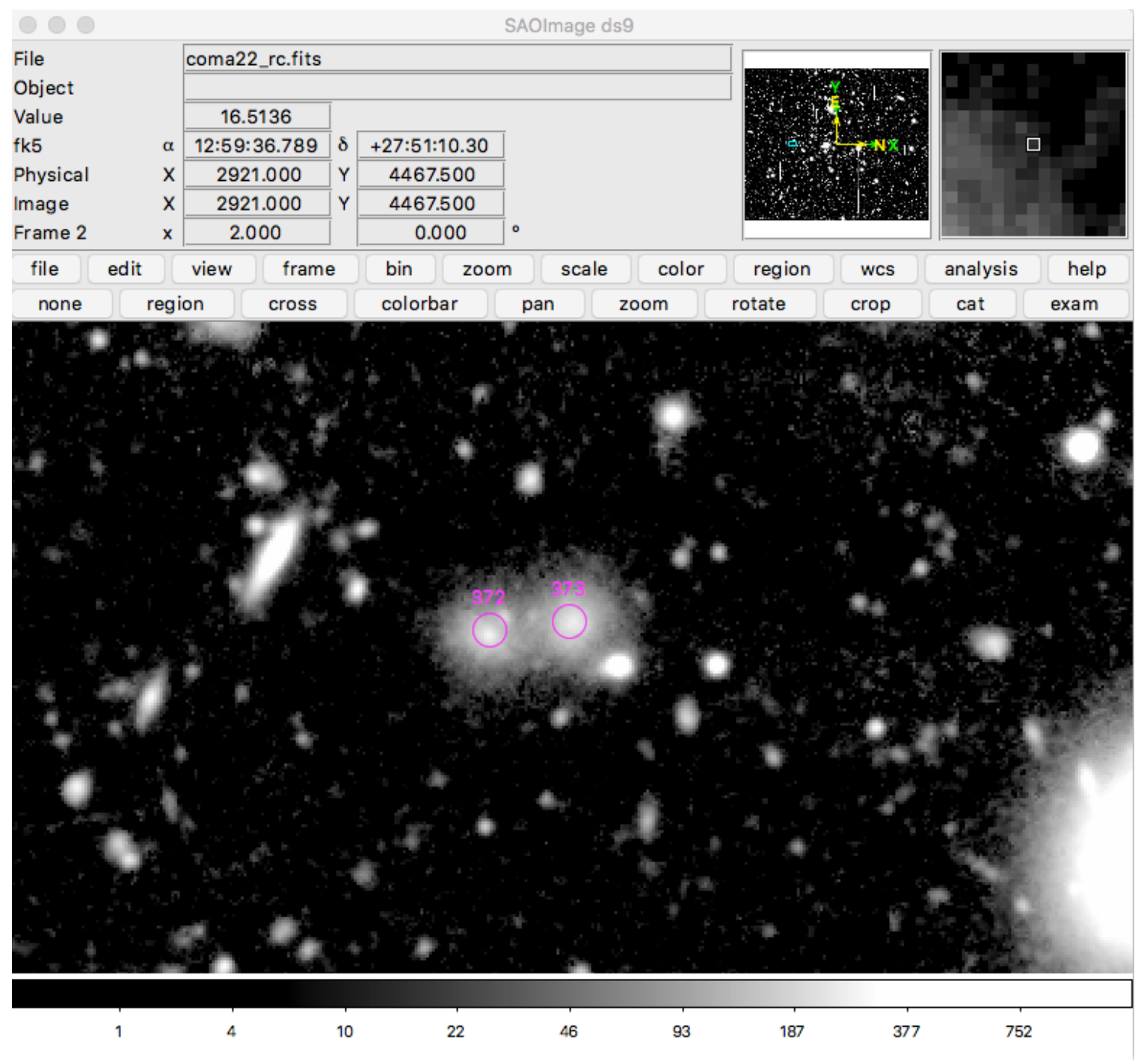

Figure 4.14: UDGs 372 and 373, a merger candidate.

Furthermore, recently the Hyper Suprime-Cam (HSC) survey started making sections of the sky available to the public. We looked through those areas for potential UDG targets. The list of these initial targets identified by eye is in Table 
4.1. Further investigation is needed to analyze the objects in this list and vet their likelihood of being a UDG.

Table 4.1: R.A. and Decl. values of candidate UDGs.

\begin{tabular}{|c|c|}
\hline R.A. (degrees) & Decl. (degrees) \\
\hline 39.143383 & -5.917902 \\
245.419242 & 43.840453 \\
245.228918 & 43.859322 \\
245.253515 & 43.858970 \\
39.1362330 & -5.731764 \\
39.1508391 & -5.666776 \\
39.1508627 & -5.666939 \\
245.360687 & 42.878641 \\
246.923511 & 42.672466 \\
246.924296 & 42.677318 \\
246.819740 & 42.652269 \\
246.826515 & 42.647801 \\
246.720036 & 42.635637 \\
246.598993 & 42.660354 \\
246.681010 & 42.695486 \\
246.673963 & 42.696189 \\
246.701826 & 42.741860 \\
246.761903 & 42.726408 \\
247.451697 & 43.887298 \\
247.406482 & 44.095291 \\
247.405366 & 44.104150 \\
247.113238 & 43.957488 \\
247.021027 & 44.014078 \\
247.026710 & 43.995645 \\
246.572766 & 43.937416 \\
246.477155 & 43.974822 \\
246.477154 & 43.974589 \\
246.404531 & 43.937284 \\
245.956935 & 43.863976 \\
246.726291 & 43.449959 \\
244.665474 & 42.983831 \\
244.637975 & 43.121248 \\
244.632729 & 43.148450 \\
243.226274 & 43.495260 \\
243.135651 & 43.731515 \\
214.836716 & 52.064413 \\
215.018353 & 52.131928 \\
215.057347 & 52.125394 \\
213.465612 & 52.740952 \\
150.546963 & 2.45450 \\
\hline & \\
& \\
\hline
\end{tabular}


Table 4.1 - Continued from previous page

\begin{tabular}{|c|c|}
\hline R.A. (degrees) & Decl. (degrees) \\
\hline 150.313000 & 2.4697 \\
\hline 150.11750 & 2.67803 \\
\hline 34.344269 & -5.496021 \\
\hline 35.449492 & -5.650721 \\
\hline 37.477228 & -5.596015 \\
\hline 37.469855 & -5.570330 \\
\hline 37.547350 & -5.557176 \\
\hline 37.634657 & -5.504200 \\
\hline 38.125192 & -5.241695 \\
\hline 38.801867 & -5.695513 \\
\hline 38.900798 & -5.573807 \\
\hline 38.894650 & -5.555482 \\
\hline 38.913426 & -5.552337 \\
\hline 39.113178 & -5.572803 \\
\hline 38.700206 & -5.323021 \\
\hline 38.644669 & -5.265620 \\
\hline 352.624837 & 0.124185 \\
\hline 352.624417 & 0.123905 \\
\hline 352.429868 & 0.315455 \\
\hline 351.400140 & 0.394337 \\
\hline 336.124775 & 1.231975 \\
\hline 38.673491 & -5.233538 \\
\hline 38.720793 & -5.150376 \\
\hline 351.20911 & 0.414361 \\
\hline 336.970639 & 0.028763 \\
\hline 336.960539 & 0.114484 \\
\hline 336.885153 & 0.652907 \\
\hline 336.842868 & 0.980939 \\
\hline 335.977205 & 1.330978 \\
\hline 335.373733 & 1.535139 \\
\hline 216.504776 & -0.314777 \\
\hline 216.516184 & -0.296905 \\
\hline 216.478220 & -0.244304 \\
\hline 216.490257 & -0.195903 \\
\hline 216.525347 & -0.166739 \\
\hline 216.484433 & -0.120230 \\
\hline 216.484316 & -0.120335 \\
\hline 216.486416 & -0.118970 \\
\hline 216.439927 & -0.104482 \\
\hline 216.410130 & -0.037432 \\
\hline 216.459010 & -0.037455 \\
\hline 216.554797 & 0.050817 \\
\hline 216.572366 & 0.059740 \\
\hline 37.570725 & -4.215436 \\
\hline 37.446170 & -4.133989 \\
\hline 336.829289 & 0.612572 \\
\hline 336.899306 & 0.928526 \\
\hline 335.824710 & 1.411616 \\
\hline
\end{tabular}


Table 4.1 - Continued from previous page

\begin{tabular}{|c|c|}
\hline R.A. (degrees) & Decl. (degrees) \\
\hline 37.411891 & -4.103388 \\
\hline 335.902881 & 1.38718 \\
\hline 335.788527 & 1.40395 \\
\hline 335.478975 & 1.50525 \\
\hline 335.480957 & 1.49396 \\
\hline 38.886121 & -5.49935 \\
\hline 244.072338 & 42.9145 \\
\hline 243.886224 & 42.92536 \\
\hline-37.629447 & -4.217085 \\
\hline 37.231062 & -4.003629 \\
\hline 37.351679 & -3.85000 \\
\hline 246.477154 & 43.97458 \\
\hline 181.768076 & 1.183734 \\
\hline 181.777932 & 1.182180 \\
\hline 181.438970 & 1.204667 \\
\hline 181.424124 & 1.205131 \\
\hline 181.134160 & 1.186923 \\
\hline 180.385939 & 1.203614 \\
\hline 179.658118 & 1.209028 \\
\hline 180.328601 & 1.197773 \\
\hline 180.071410 & 1.202252 \\
\hline 179.658118 & 1.209028 \\
\hline 179.895816 & 1.202381 \\
\hline 179.864160 & 1.206238 \\
\hline 179.466778 & 1.203636 \\
\hline 179.393327 & 1.207906 \\
\hline 178.894815 & 1.146118 \\
\hline 179.173917 & 0.918338 \\
\hline 179.148920 & 0.959871 \\
\hline 179.895839 & 1.202380 \\
\hline 179.063537 & 1.001596 \\
\hline 179.916840 & 1.197569 \\
\hline 179.935274 & 1.198685 \\
\hline 179.400945 & 0.799234 \\
\hline 179.587534 & 0.771581 \\
\hline 179.530485 & 0.758588 \\
\hline 179.496517 & 0.152247 \\
\hline 179.805531 & 1.008733 \\
\hline 179.199664 & -0.440498 \\
\hline 179.286180 & -0.246422 \\
\hline 179.433742 & -0.862808 \\
\hline 179.442857 & -0.998557 \\
\hline 179.470209 & -1.034198 \\
\hline 179.134258 & -1.309309 \\
\hline 179.342295 & -1.313434 \\
\hline 179.468455 & -1.204144 \\
\hline 179.518694 & -1.072497 \\
\hline 179.112793 & -1.311393 \\
\hline
\end{tabular}


Table 4.1 - Continued from previous page

\begin{tabular}{|c|c|}
\hline R.A. (degrees) & Decl. (degrees) \\
\hline 179.089432 & -1.364220 \\
178.133081 & -1.096178 \\
177.923592 & -0.921566 \\
177.641726 & -0.790413 \\
177.503941 & -0.689971 \\
177.532643 & -0.597038 \\
\hline
\end{tabular}

\subsubsection{UDG candidate example}

Here we present an example of assessing the size of a possible UDG in an attempt to further vet whether it is likely to be an ultra-diffuse galaxy. An example of this preliminary analysis was done for a very-faint object in between two apparently bigger galaxies. It is portrayed in Figures 4.15, 4.16, and 4.17 at different zoom levels both through the HSCMAP and the HSCMAP2 editions of the Hyper Suprime-Cam data web visualizations. Its R.A. and Decl. coordinates are 246.477154, 43.974589 degrees. A search through the SDSS DR13 Navigate tool revealed its database identification number as 1237655474503483537 . A search in the NASA Extragalactic Database did not produce any matches for this SDSS ID. 


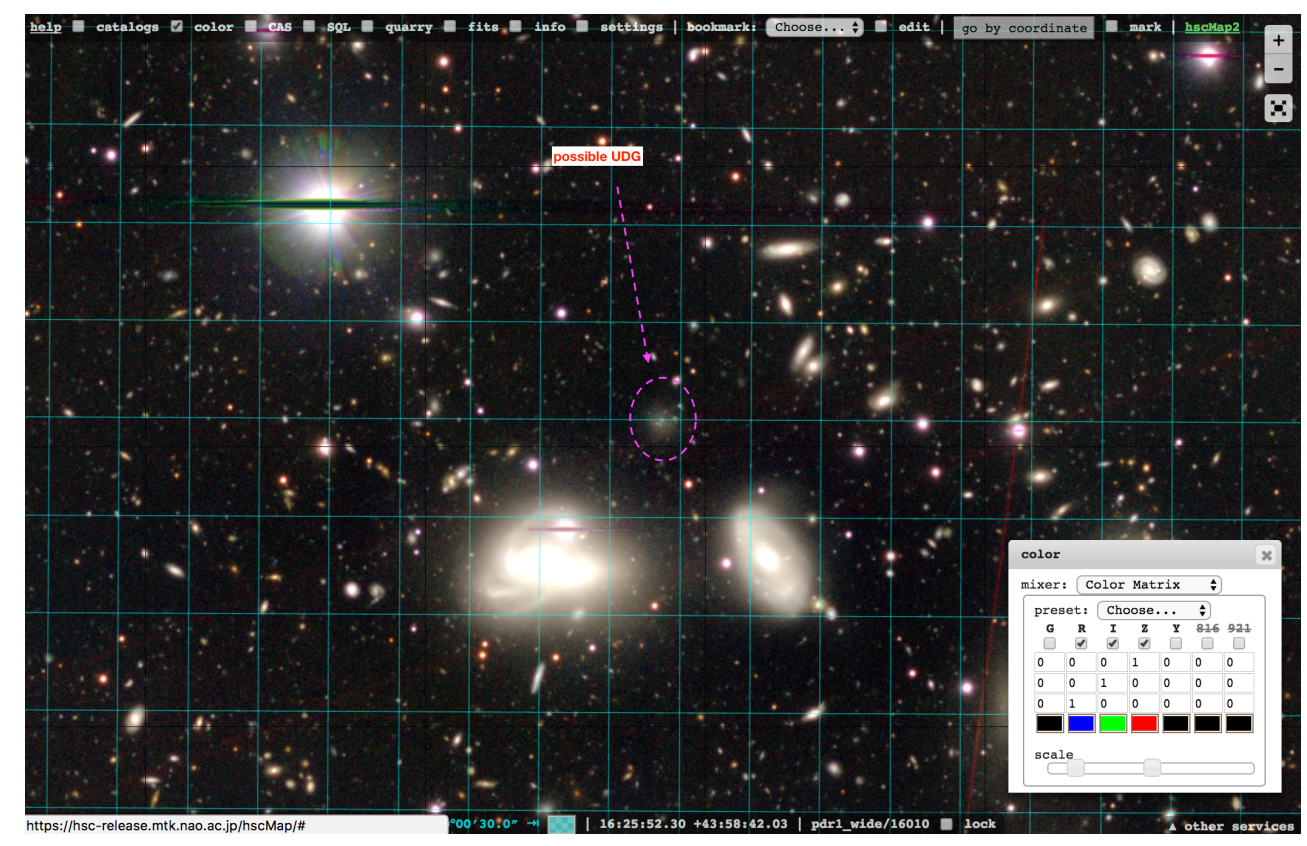

Figure 4.15: Candidate UDG on the HSCMAP viewer with its immediate environment.

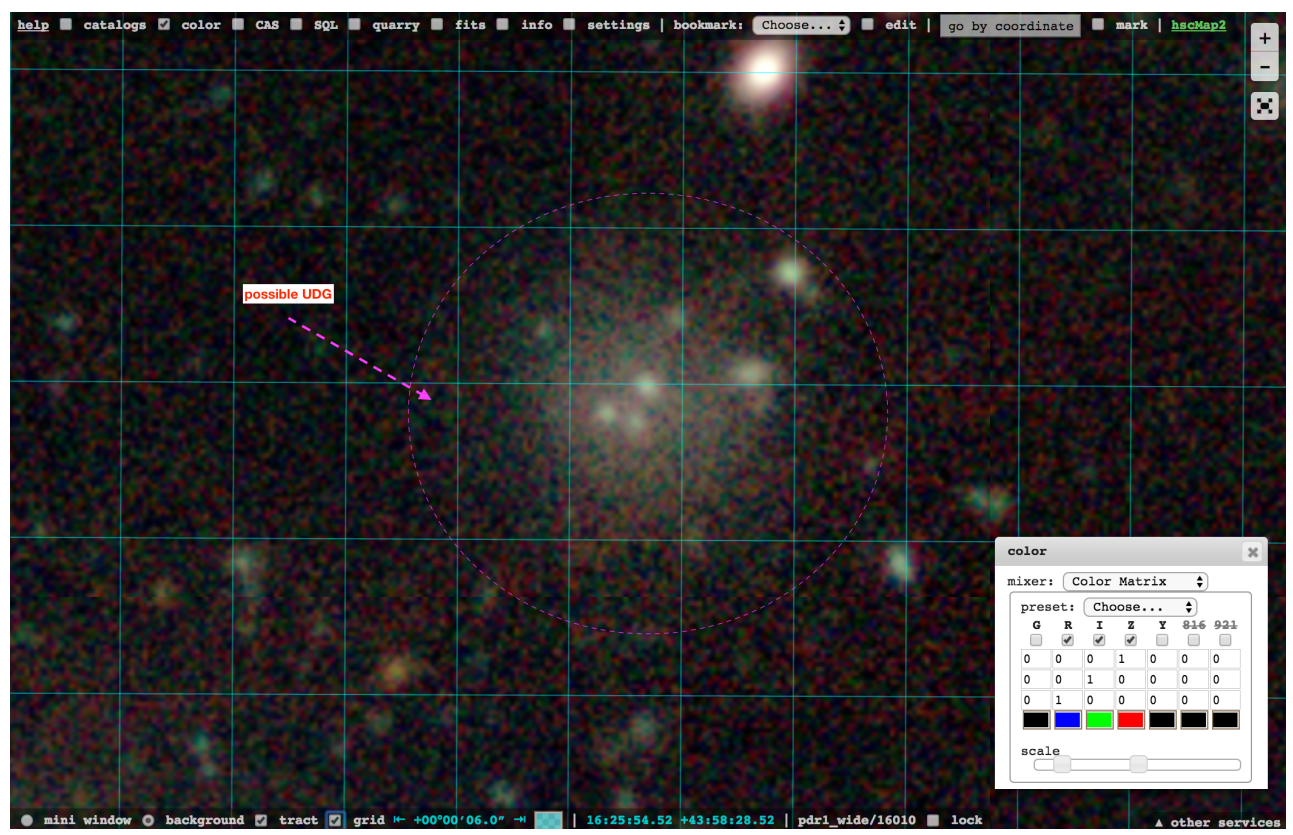

Figure 4.16: A zoomed-in image of the candidate UDG on the HSCMAP viewer. 


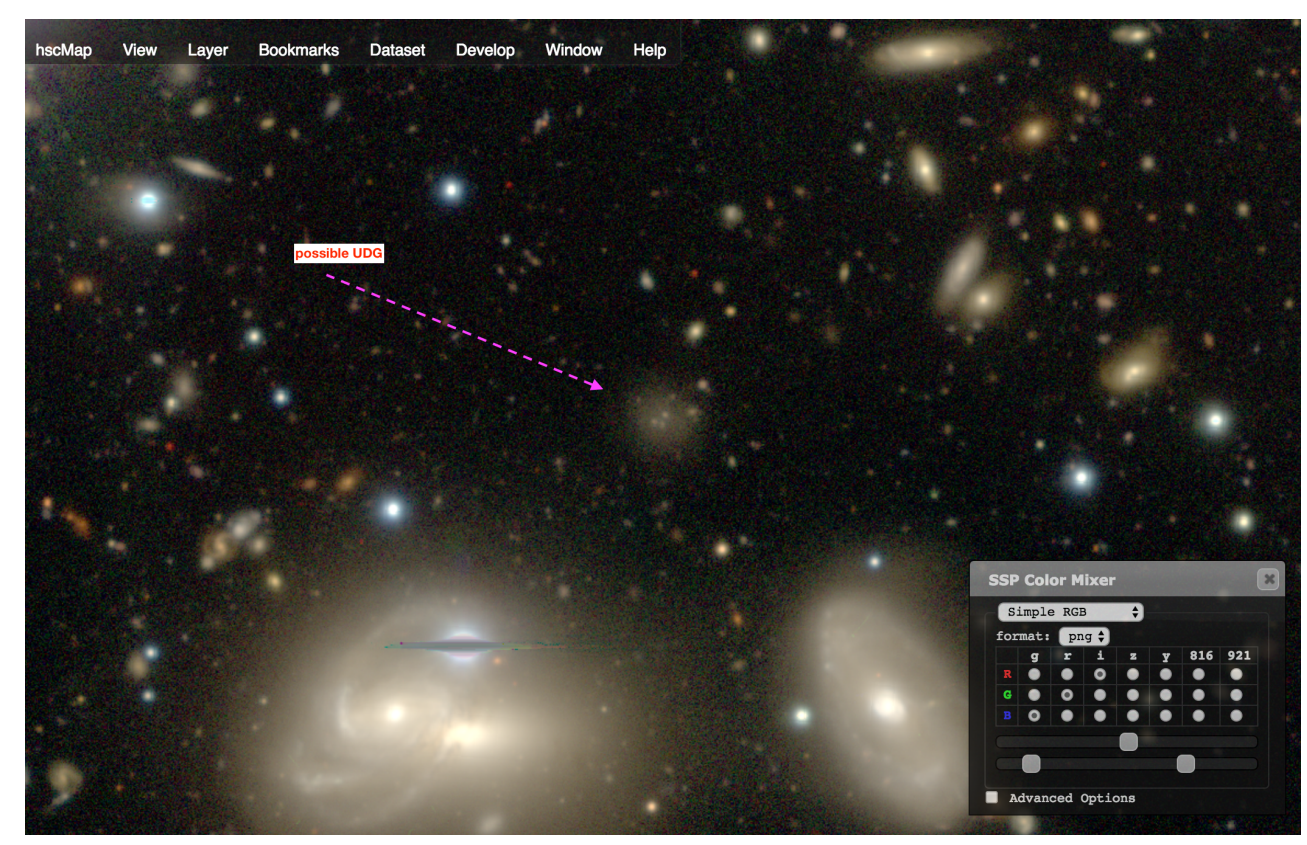

Figure 4.17: Candidate UDG in the HSCMAP2 visualization.

After visual identification, we used the approximate border coordinates from the HSCMAP to get the apparent diameter (by eye) and obtained $\theta=11.09$ arcsec. The two gigantic galaxies nearby were roughly at $z=0.03$ from the SDSS measurements. Their SDSS identification numbers were 1237655474503483507 and 1237655474503483504. We used $H_{0}=73 \mathrm{~km} \mathrm{~s}^{-1}$ to calculate the distance to the object, resulting in a distance of $123.3 \mathrm{Mpc}$. We used the physical size to apparent angle relation to obtain an estimate of the radius of the object, $3.32 \mathrm{kpc}$. The resultant radius was within the range of a typical UDG size. Thus, this is an interesting object for spectroscopic follow-up to confirm whether or not this object is a true UDG. Possible globular clusters embedded within the image of this potential UDG object were observed as bright patches overlaid on the galaxy. 


\subsection{Transformations pipeline}

We developed a pipeline of transformations and linear fits to obtain the SDSS $g-i$ color and $r$ magnitude for temperature and luminosity axes on the color-magnitude diagram for UDGs. The Subaru $B-R$ colors are not the same as the standard $B-R$ colors. We calculated the offset relationship for the Subaru $B-R$ color for UDGs in the Yagi et al. (2016) catalog by converting the Subaru $B-R$ color to the normal $B-R$ color according to equation 3.3.

Since the UDGs were observed by the Suprime-Cam instrument of the Subaru telescope, the measurements of colors and magnitudes needed to be converted to the SDSS system to be able to compare them to the normal galaxies in the Coma cluster. We carried out a linear fit analysis for color-color derivations and applied the transformation equations from the methodology section to obtain the SDSS $r$ magnitude values.

First, a linear fit was performed to determine the transformation relationship between the standard $B-R$ and the SDSS $g-i$ colors for normal galaxies in the Coma cluster. Our result was a relationship between the standard $B-R$ color and the SDSS $g-i$ color for the normal galaxies in the Coma cluster. While the Consolandi et al. (2016) paper provided R.A. and Decl. values for the member galaxies of the Coma cluster, it only gave the SDSS $g$ and $i$ magnitudes for those galaxies. Since we also needed at least the SDSS $r$ magnitude, we used the CrossID tool summarized in Methods earlier to obtain the missing SDSS $r$ magnitude. Then, the $B-R$ color was obtained using equation 3.7. Figure 4.18 shows the linear fit relationship between $B-R$ and $g-i$ colors graphically. 


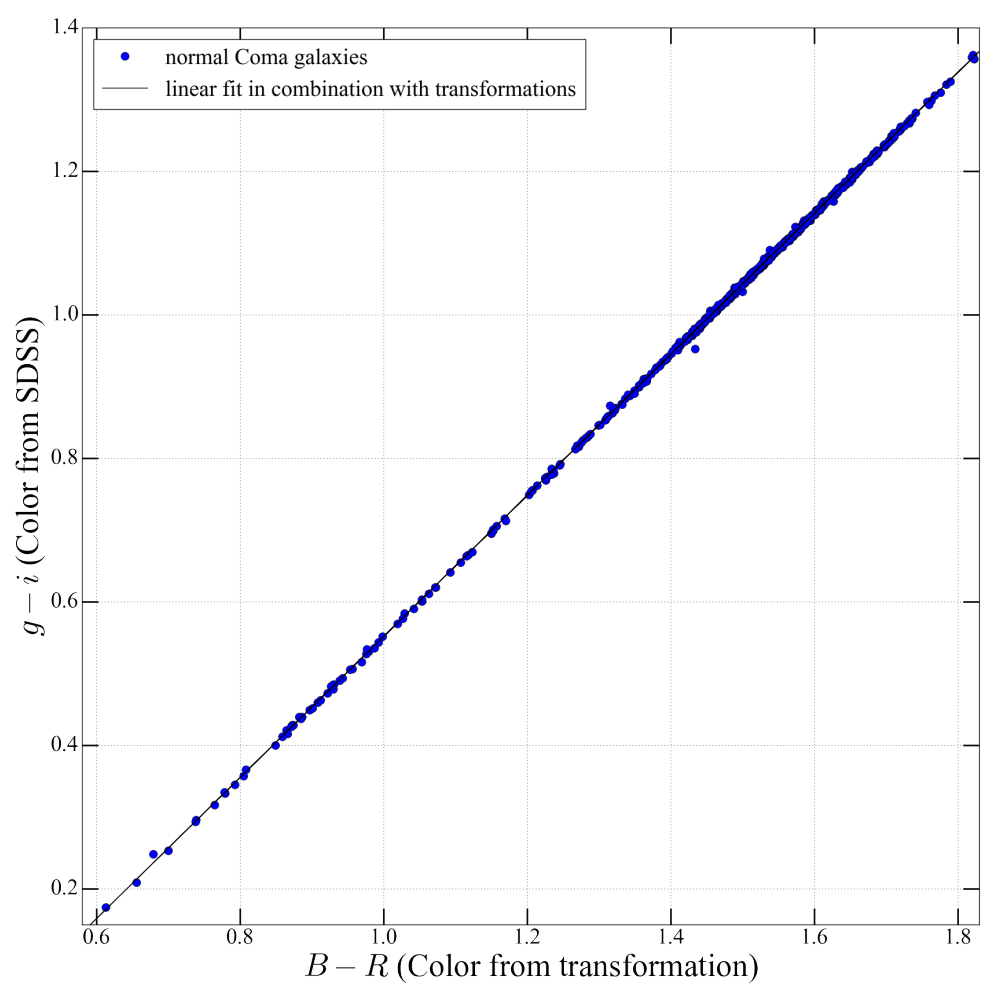

Figure 4.18: Standard $B-R$ to the SDSS $g-i$ transformation for normal galaxies.

The linear fit was performed using the python scipy.stats.linregress algorithm. We did not observe any obvious outliers. Recall that the galaxies in Figure 4.18 were normal galaxies in the Coma cluster whose membership in the cluster was confirmed via the Consolandi et al. (2016) catalog. The linear fit from Figure 4.18 is expressed in the following equation:

$$
g-i=0.98273 \times(B-R)-0.43093
$$

The fit expressed by equation 4.1 was obtained by using the data for only normal galaxies in the Coma cluster. The total number of these galaxies was 669 . Their positions were within the apparent radius of 1.5 degrees from the central 
coordinates of Coma (R.A. and Decl. of $194.9531 \mathrm{deg}, 27.9807 \mathrm{deg}$ ). We obtained these coordinates from the NED extragalactic database. The relationship given by equation 4.1 was used to derive the SDSS $g-i$ colors of UDGs after converting the Subaru $B-R$ colors to the normal $B-R$ colors via equation 3.3.

Next, another linear fit was employed to determine the SDSS $g-i$ vs. $g-r$ correlation for normal galaxies in the Coma cluster, again in combination with the previously carried out transformations. Our next result is the linear fit equation as follows:

$$
(g-r)=0.6595 \times(g-i)+0.0125
$$

The color-color plot is depicted in Figure 4.19. It depicts the relation between the SDSS $g-i$ and $g-r$ colors using data for normal galaxies in the Coma cluster. The solid line represents the linear fit of the color-color plot. We used this result in combination with the derived SDSS $g-i$ colors (see equation 4.1 ) to get the SDSS $g-r$ colors for these galaxies. 


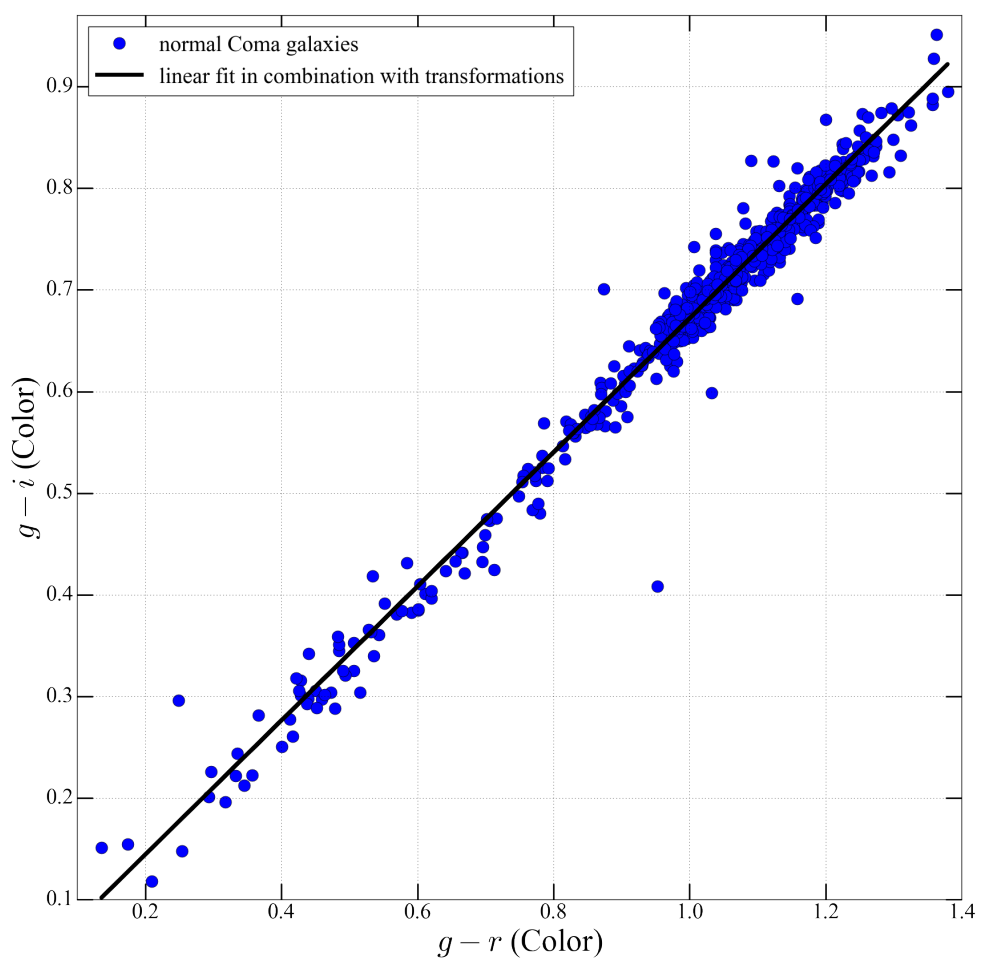

Figure 4.19: $g-i$ vs. $g-r$ color-color relation

Our next result was the calculation of the SDSS $r$ magnitudes for UDGs, the final piece of the color-magnitude diagram puzzle for UDGs. We started with the available data on the Subaru $R$ magnitude measurements from the Yagi et al. (2016) catalog. The Subaru $R$ magnitude was converted to the SDSS $g$ magnitude via equation $3.9, g=R+0.8$. Then, we used equations 3.10, $r=g-(g-r)$, to obtain the SDSS $r$ magnitude for UDGs by substituting equation 3.9 for $g$ magnitude and the derived SDSS $g-r$ color from equation $4.2(g-r)=0.6595 \times(g-i)+0.0125$. Thus, through the above steps in the analysis pipeline, we had both the SDSS $g-i$ color to represent temperature and the SDSS $r$ magnitude to represent luminosity to construct the CMD plots in a convenient standard system of the SDSS ugriz magnitudes for further analysis of the enigmatic ultra-diffuse galaxies. 


\subsection{Color-magnitude diagrams}

In this section the results of color-magnitude diagrams are presented for inner and outer populations of normal and ultra-diffuse galaxies in the Coma cluster. The inner and outer populations were separated using the right ascension and declination (R.A. and Decl.) coordinates. The positions of galaxies from the Coma cluster within a 1.5 deg radius relative to the center were plotted in Figure 4.20. The coordinates were given by the Consolandi et al. (2016) catalog. The central coordinates of the Coma cluster were obtained from the NASA extragalactic database $(\mathrm{NED})$ as R.A. and Decl. $=(194.9531,27.9807)$ in degrees. The total number of these galaxies within the specified radius was 669 .

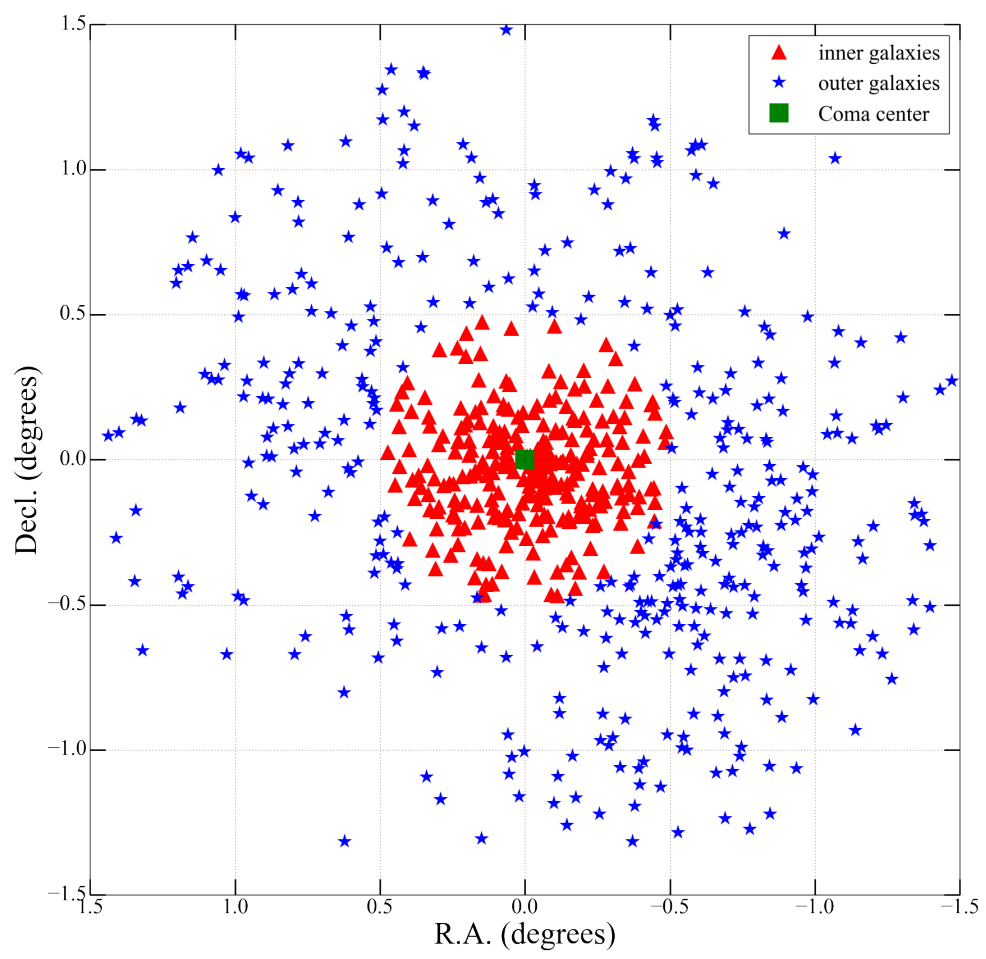

Figure 4.20: Relative positions of normal member galaxies in the Coma cluster. 
The galaxies were further subdivided into inner and outer populations based on their apparent angular distance from the cluster center. The inner galaxies were within 0.5 degrees of the center. The outer galaxies occupied the area between 0.5 degrees and 1.5 degrees from the center. The number ratio of inner normal galaxies to outer normal galaxies based on the available data was 0.689 .

Similarly, UDGs were plotted in Figure 4.21. The first plot shows all 854 UDGs. The second plot shows the 232 UDGs which had the Subaru magnitudes and color measurements available. 


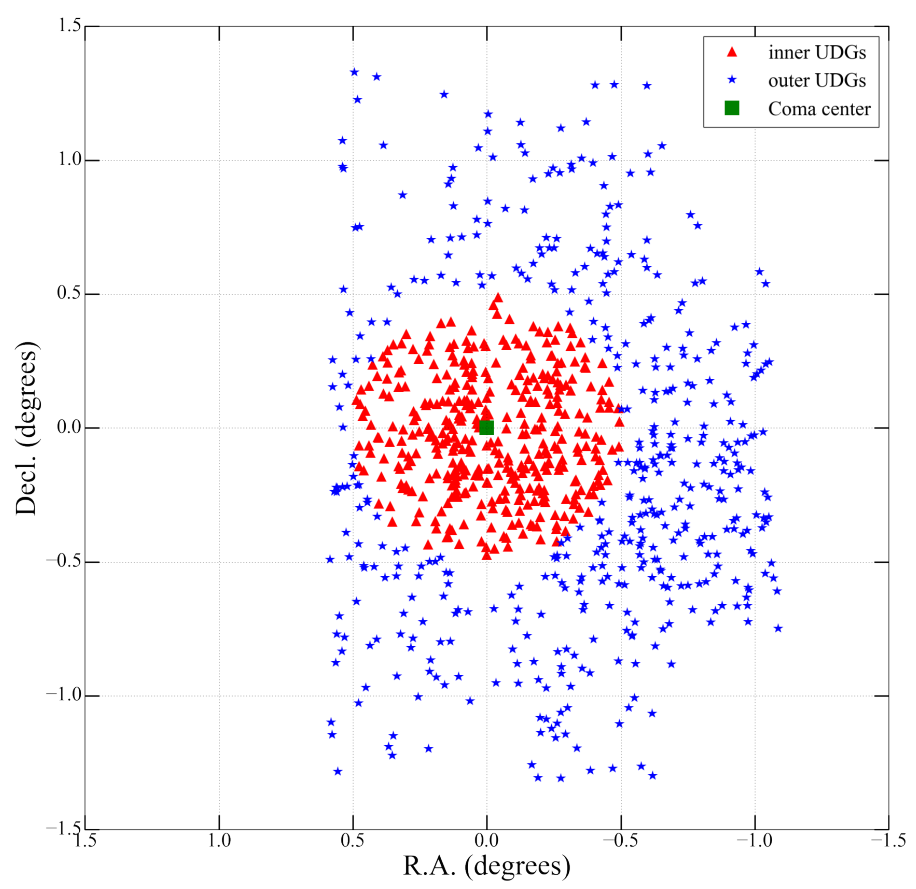

(a)

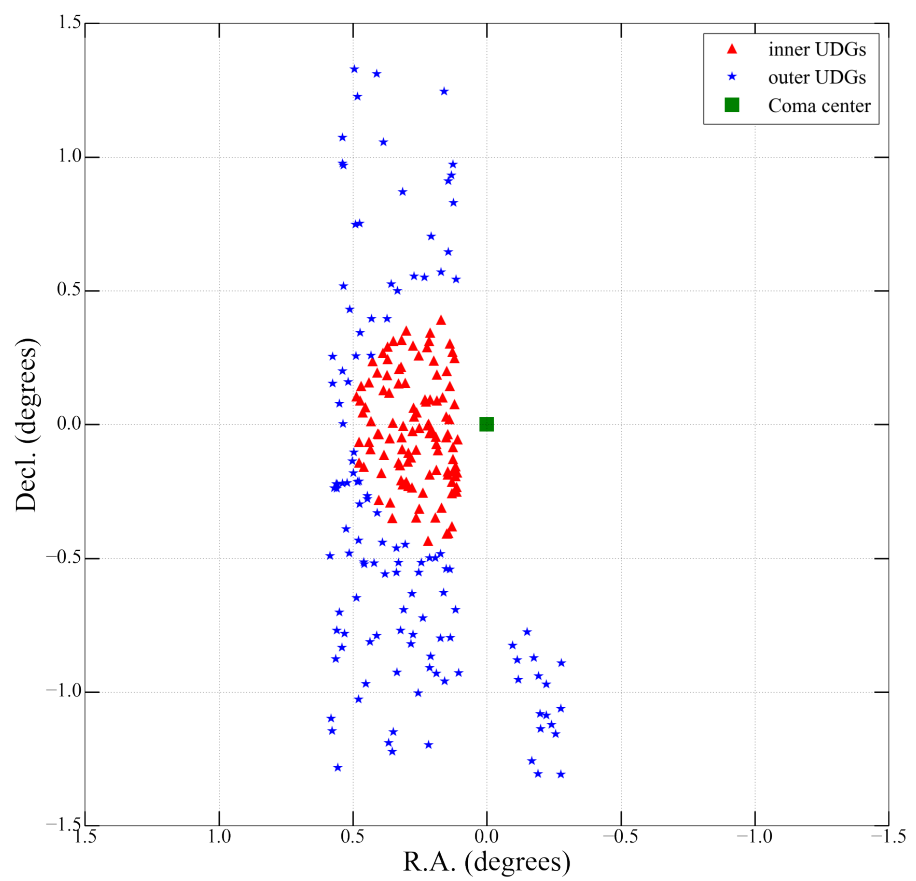

(b)

Figure 4.21: Map of (a) all UDGs and (b) UDGs with Subaru $B-R$ data. 
They were separated into inner and outer populations in an equivalent way to the normal galaxies. The ratio of inner to outer UDG numbers was higher than that for the normal galaxies and was equal to 0.825 . Note that there were blank regions in this R.A./Decl. plot because some of the areas of the sky had not been surveyed for UDGs. Thus, the comparison of the ratios of inner to outer UDGs is not robust and may change as more observations of UDGs are made, especially in the outskirts of the cluster (sampling bias).

\subsubsection{Color-magnitude diagrams of normal galaxies}

In this section we present the color-magnitude diagram of inner and outer populations of normal galaxies in the Coma cluster. The CMD plots use the reversed axis convention according to which the brightness increases from left to right and thus the magnitude values decrease in the same direction.

The linear fits of color-magnitude trends for inner and outer populations of normal galaxies in the Coma cluster are presented in Figure 4.22. Note that the galaxies with larger $g-i$ color are grouped in an obvious band. It is known as the "red sequence" of galaxies. On the other hand, the scattered galaxies with lower

$g-i$ values are termed the "blue cloud". Indeed, in optical wavelengths, galaxies with larger colors typically appear to be redder. 


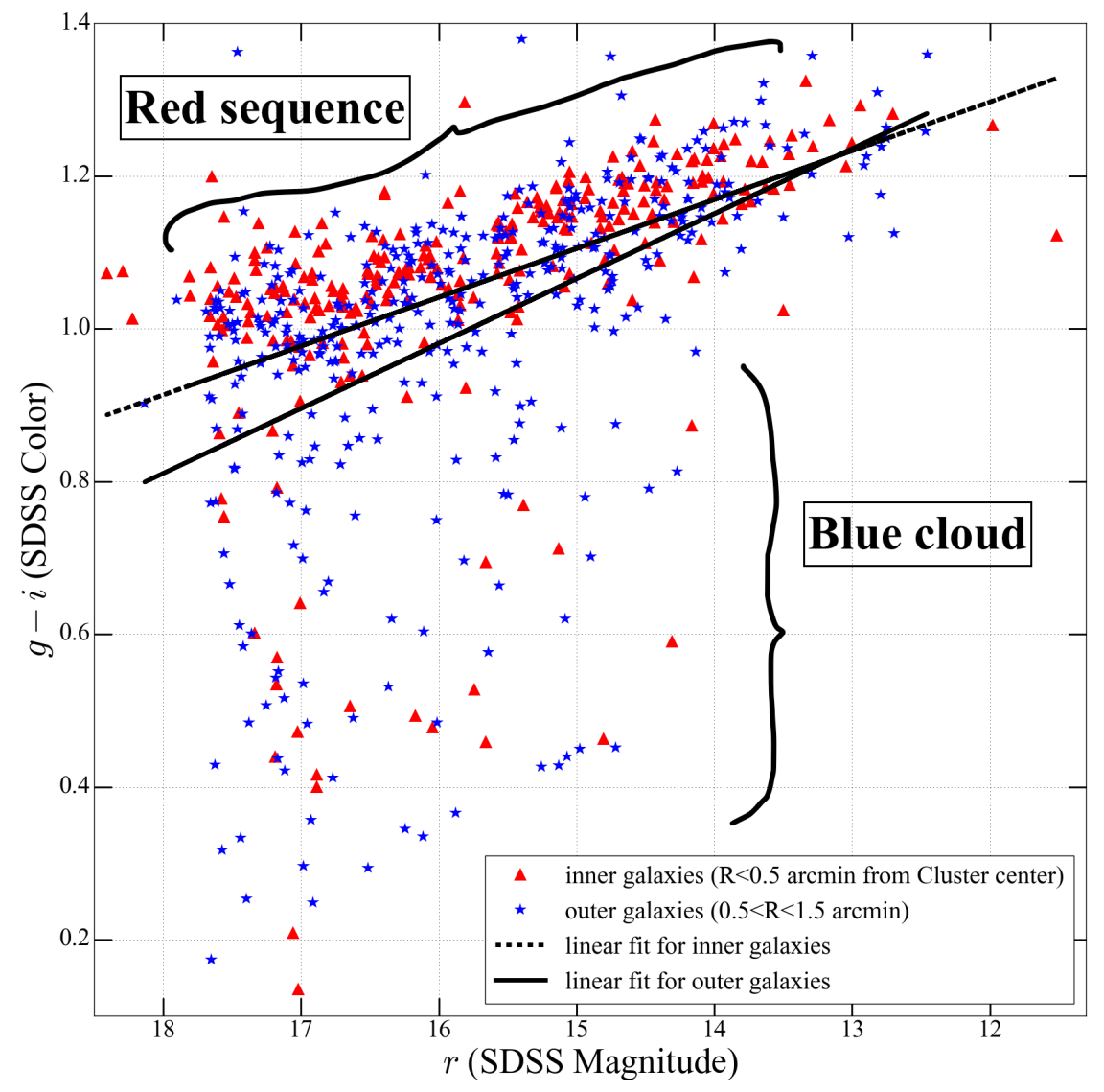

Figure 4.22: CM trends of inner and outer populations of normal galaxies.

The equation for linear fit of inner galaxies is:

$$
g-i=-0.06395 \times r+2.06441
$$

The following relation describes the linear fit for outer galaxies:

$$
g-i=-0.08501 \times r+2.34073
$$

However, these were not robust linear fits because this model was affected by the objects in the blue cloud. A more robust fitting ignoring most of the blue cloud galaxies resulted in a similar offset between the inner and outer populations of 
normal galaxies. To disregard the blue cloud outliers for the red sequence fitting, we used equations 4.5 and 4.6 as a first approximation of the fit. Then, we adopted a limiting lower boundary of $\Delta g-i=-0.2$ from the first approximation for both the inner and outer populations following the Yamanoi et al. (2012) relation for the red sequence width. All of the galaxies below that boundary were ignored in the second fitting, resulting in a more precise set of models expressed in Figure 4.23.

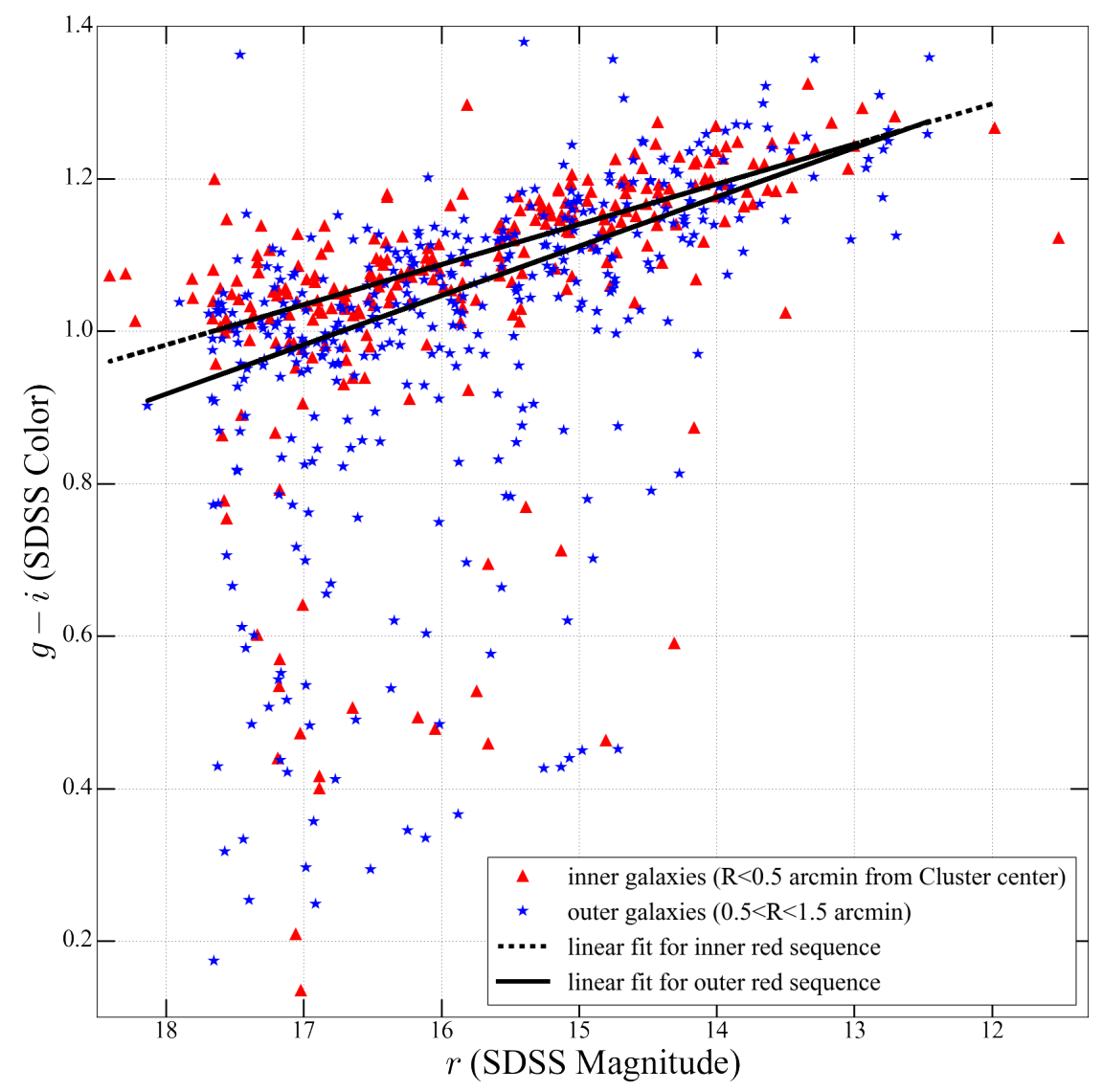

Figure 4.23: CM trends of inner and outer red sequences.

The equation for linear fit of inner red sequence is:

$$
g-i=-0.05270 \times r+1.93039
$$


The following relation describes the linear fit for outer galaxies:

$$
g-i=-0.06459 \times r+2.07994
$$

This result showed that the red sequences of inner and outer populations of normal galaxies in the Coma cluster were offset by about $g-i=0.15$ color width.

\subsubsection{Color-magnitude diagrams of UDGs}

In this section, we present the color-magnitude diagrams of inner and outer populations of ultra-diffuse galaxies in the Coma cluster. The general fit for the UDGs in the color-magnitude diagram had a slope which was not the same as the

slope of the red sequence trend by Yamanoi et al. (2012), as shown in Figure 4.24 by a thickened line. Note that in this case the numerical value of the slope approaches zero. This trend is quantified by the following equation:

$$
(B-R)=-0.00207 \times(R)+0.94029
$$




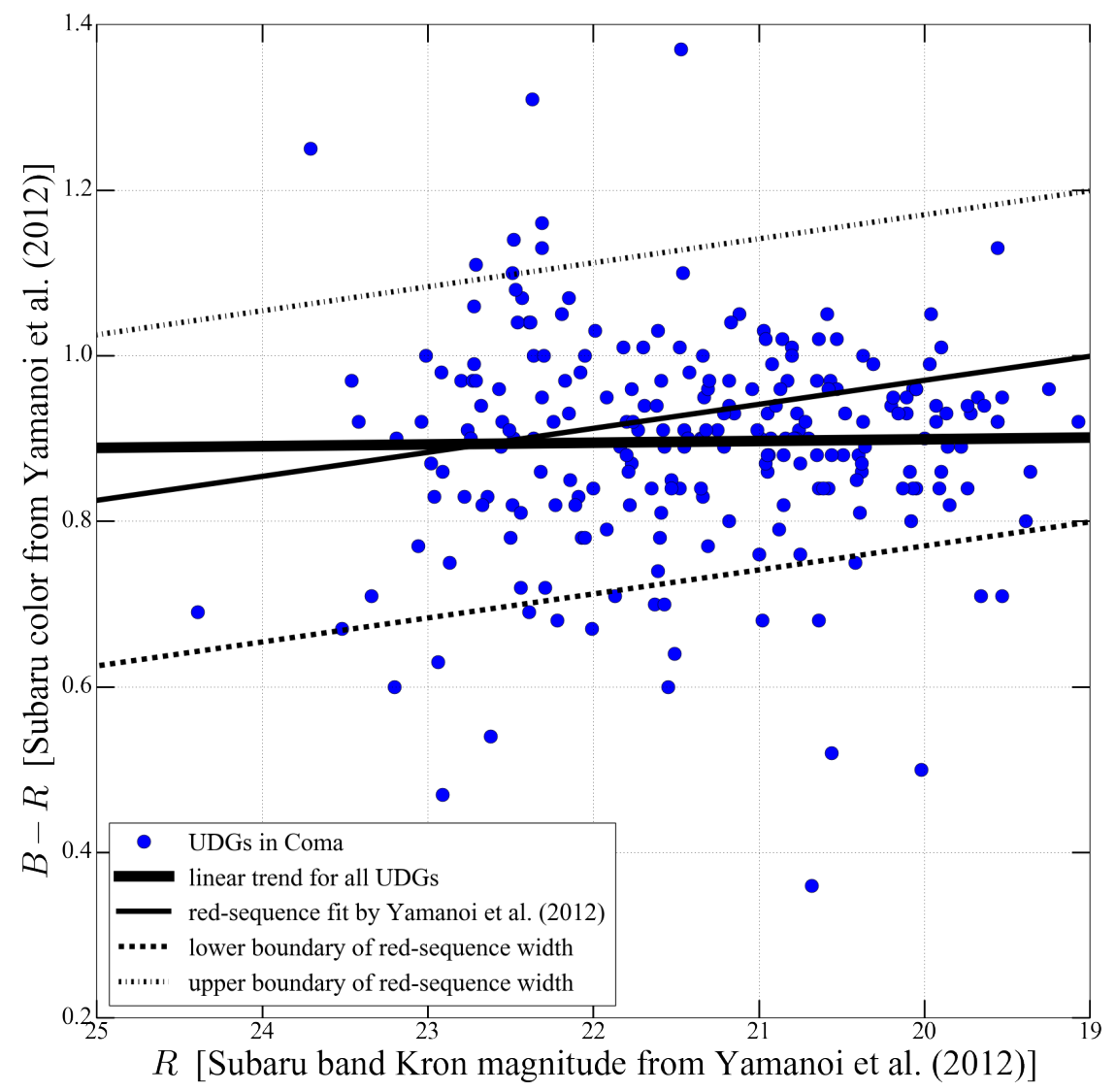

Figure 4.24: CMD trend of all UDGs.

The trends and distribution of inner and outer populations of ultra-diffuse galaxies are shown in Figure 4.25. There is a visual offset between the two trends. The inner and outer DF UDGs are portrayed using squares and circles on the same figure. 


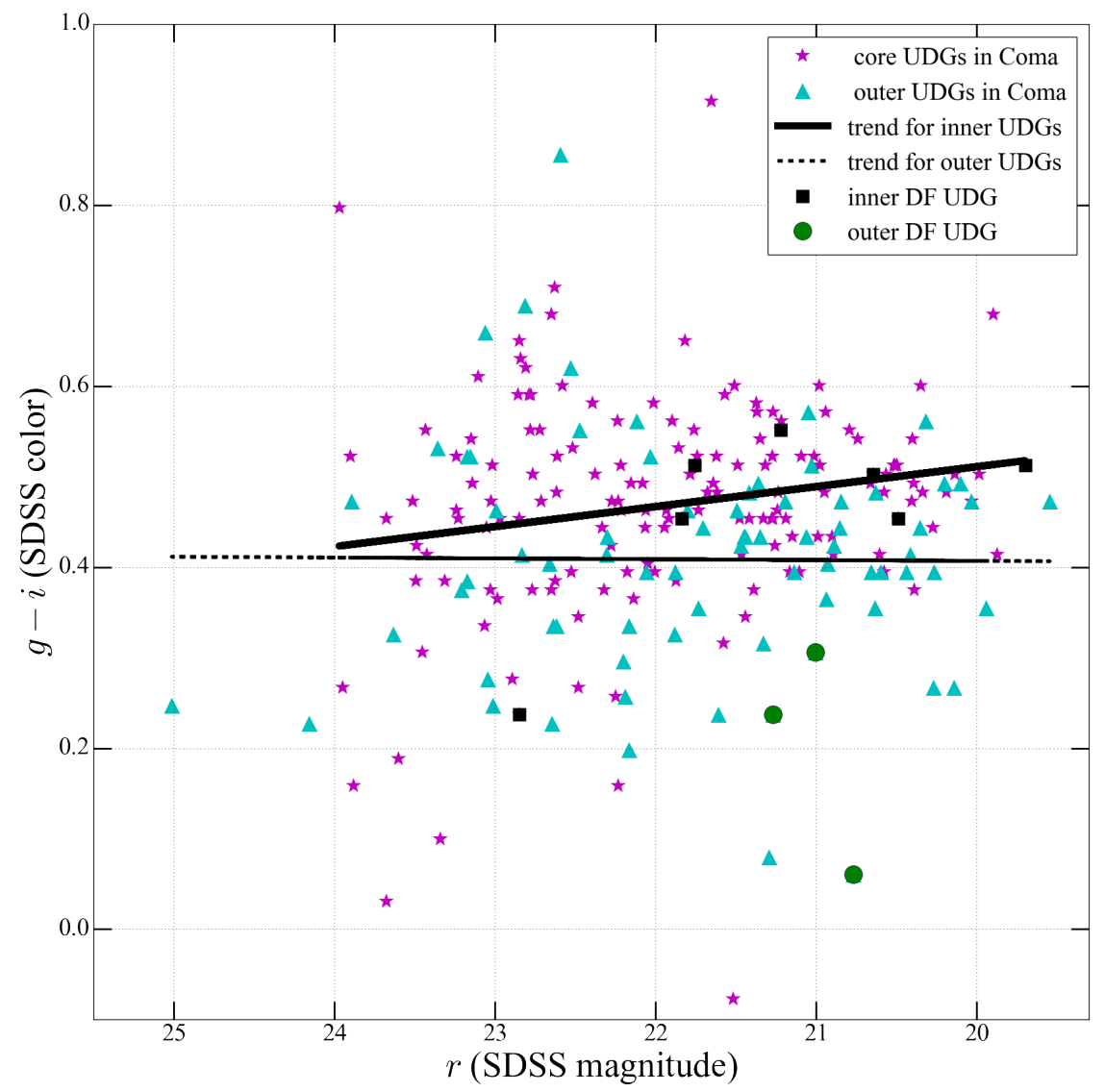

Figure 4.25: CMD trends for inner and outer UDGs.

The linear trend for inner UDGs is given by the following equation:

$$
g-i=-0.02208 \times r+0.95278
$$

The linear fit for outer UDGs is below:

$$
g-i=0.00092 \times r+0.38871
$$

Figure 4.25 also shows the original Dragonfly UDGs on the same plot for comparison. The largest offset $\Delta g-i$ between these two trends is about 0.6 , which is about four times larger than the offset between the inner and outer red sequence 
trends of normal galaxies. We also obtained a color-magnitude diagram which combined both normal and ultra-diffuse galaxies in the Coma cluster which is shown in Figure 4.26. This diagram also includes the inner and outer DF UDGs.

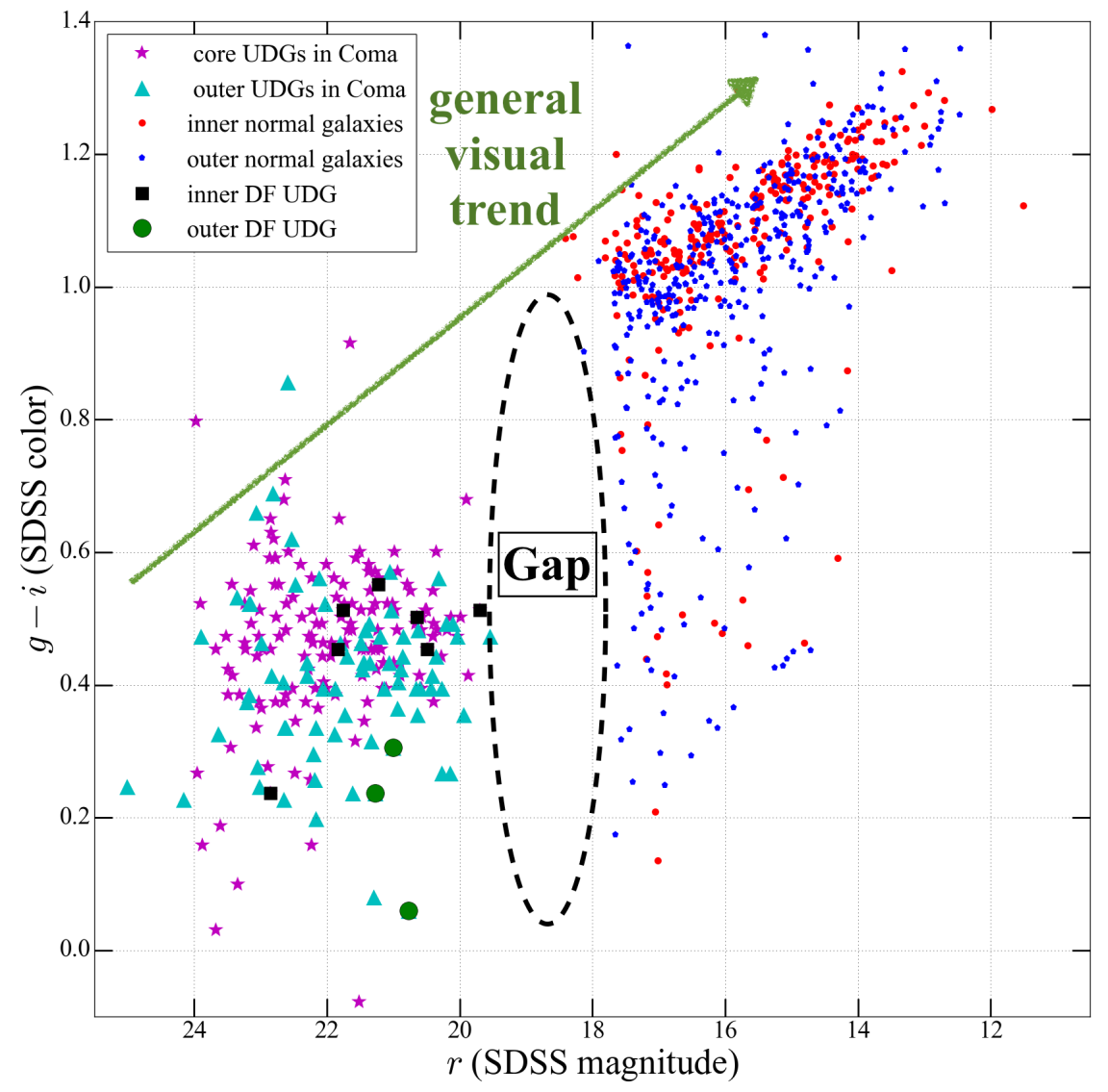

Figure 4.26: Color-magnitude diagram of all Coma cluster galaxies.

There is an obvious gap between the UDGs and the normal galaxies. UDGs also have a larger scatter associated with them, compared to the red sequence of the normal galaxies. However, the general trend between the two populations is apparent and probably signifies an evolutionary link.

Our results disagreed with the average $g-i=0.8$ as reported in the van Dokkum et al. (2015a) discovery paper. Our average $g-i$ color was between 0.4 
and 0.6. We tried an alternative way to determine the SDSS $g-i$ color using Nobu Okabe's unpublished catalog of the Coma22 field (priv. comm.). However, the fits were far from optimal between Okabe's color and the SDSS color as seen in Figure 4.27. Furthermore, the color-magnitude diagram with the SDSS parameters resulted in a very scattered plot, especially for UDGs, as shown in Figure 4.28.

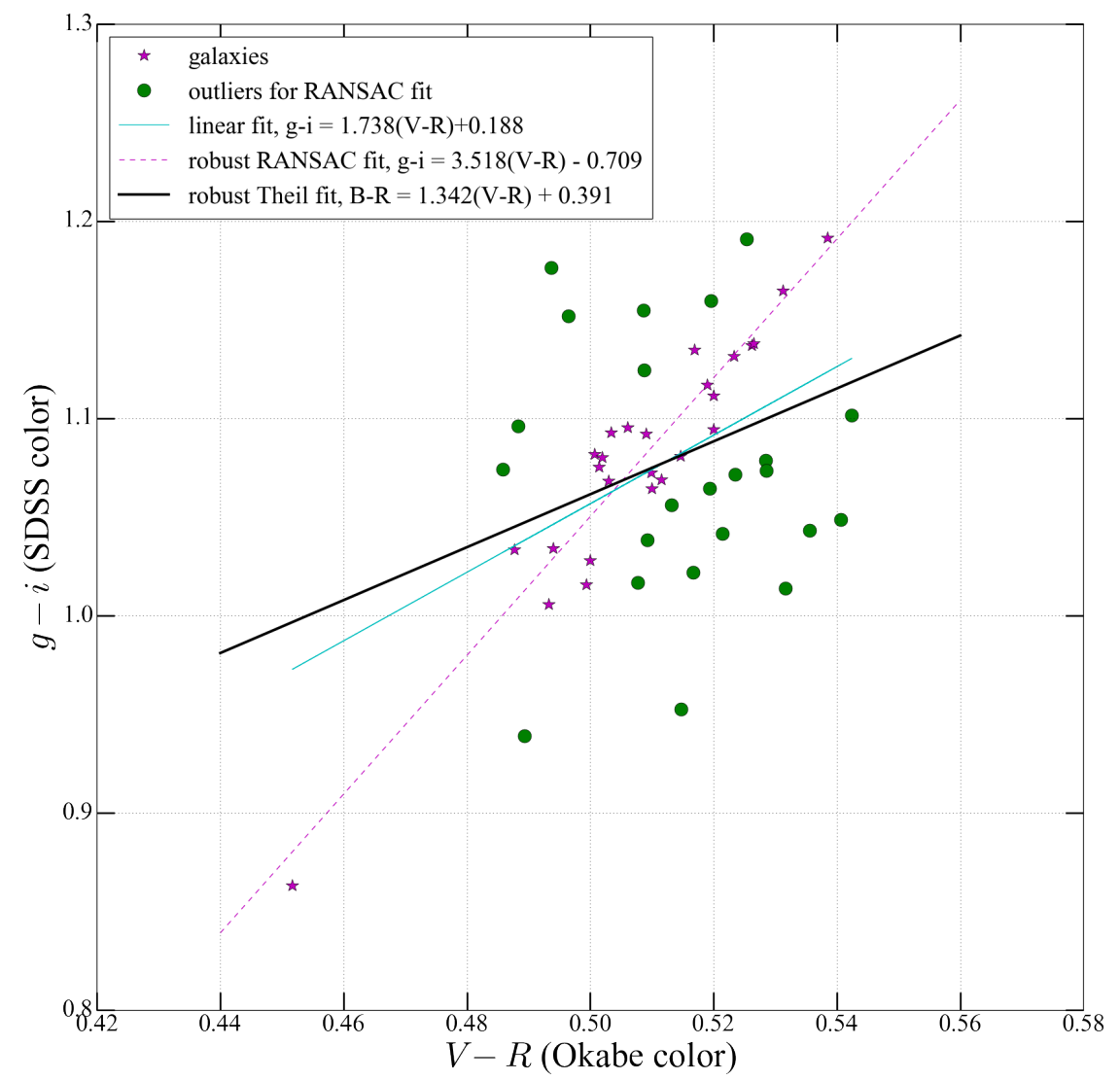

Figure 4.27: Alternative color-color relations for obtaining the SDSS $g-i$ color. 


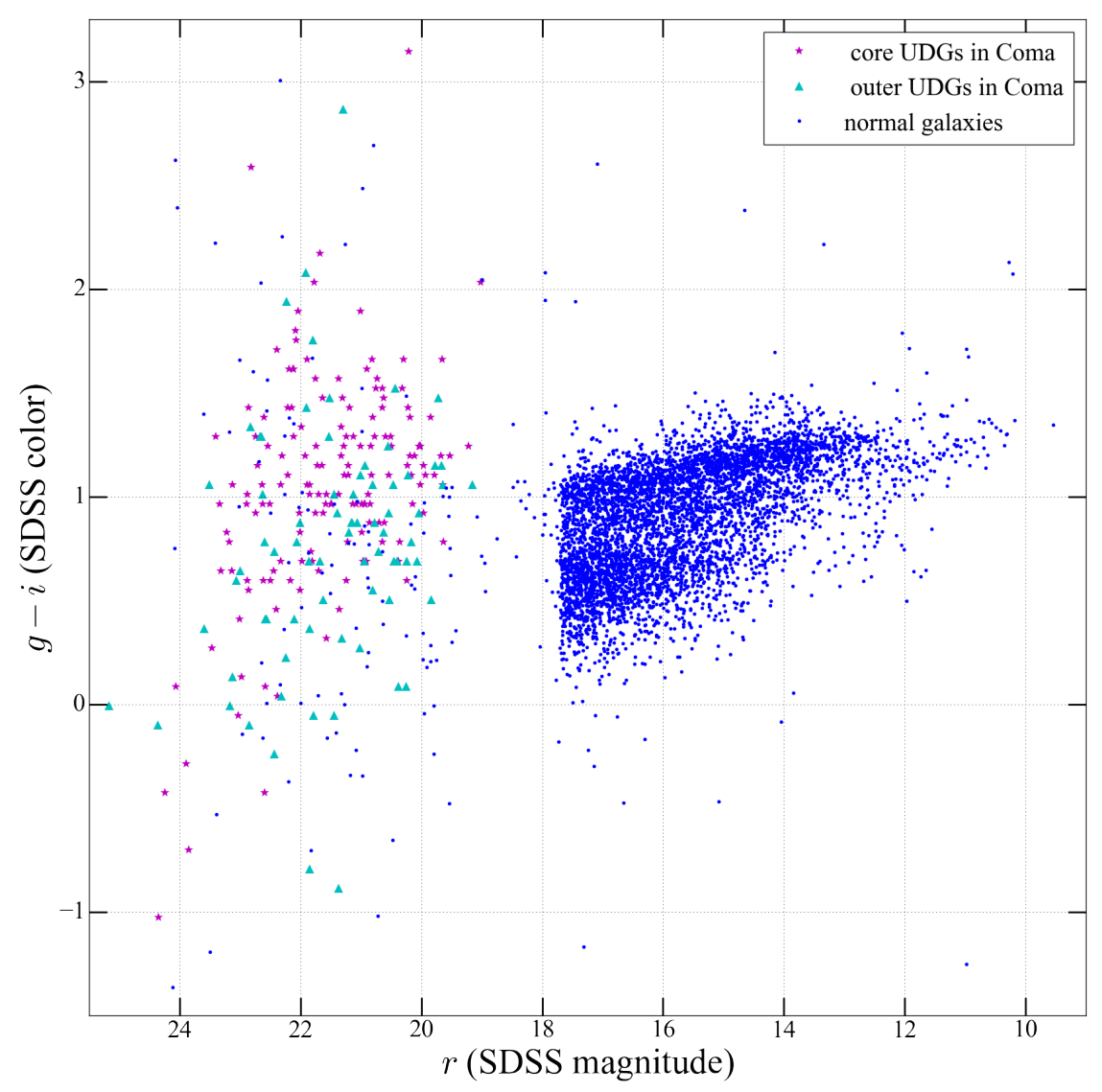

Figure 4.28: CMD with UDGs and normal galaxies using Okabe catalog.

\subsection{Stellar populations}

The beautiful aspect of obtaining the colors is that this is a segue to stellar population analysis. Colors result from different combinations of stellar populations. The significance here is the fact that since the Coma cluster galaxies are so far away it is difficult to obtain light from individual stars. Thus, models are used to interpret the combined light from these galaxies.

We used the Flexible Stellar Population Synthesis version 3.0 (Conroy et al. 2009; Conroy \& Gunn 2010) modeling package to assess what this model reveals about the constituent stars in ultra-diffuse galaxies. The exploratory plot in Figure 
4.29 showed different tracks in color-age space that stellar populations with varying metallicities $(Z)$ follow, given Padova isochrones and the BASEL spectral library. The metallicity in Figure 4.29 had units of $\left[\log \left(Z / Z_{\odot}\right)\right]$. The stellar populations were initialized by first constructing simple stellar populations with varying initial metallicity values and with the Calzetti et al. (2000) dust model.

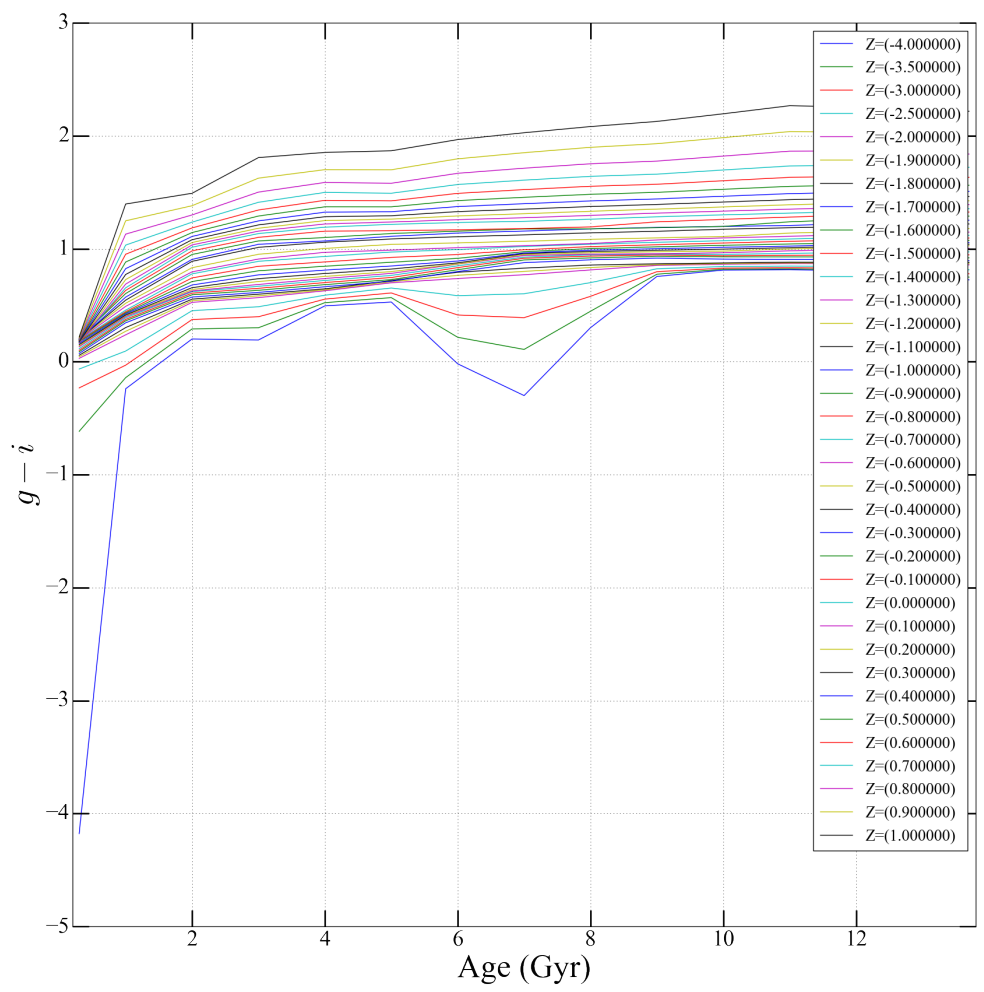

Figure 4.29: Exploratory color-age space from FSPS models.

Next, we obtained the same plot but for a particular value of metallicity: one-tenth of the metallicity of the Sun. The same settings were used for the isochrones, the spectral library, and the dust model. The resulting plot is illustrated by Figure 4.30 . 


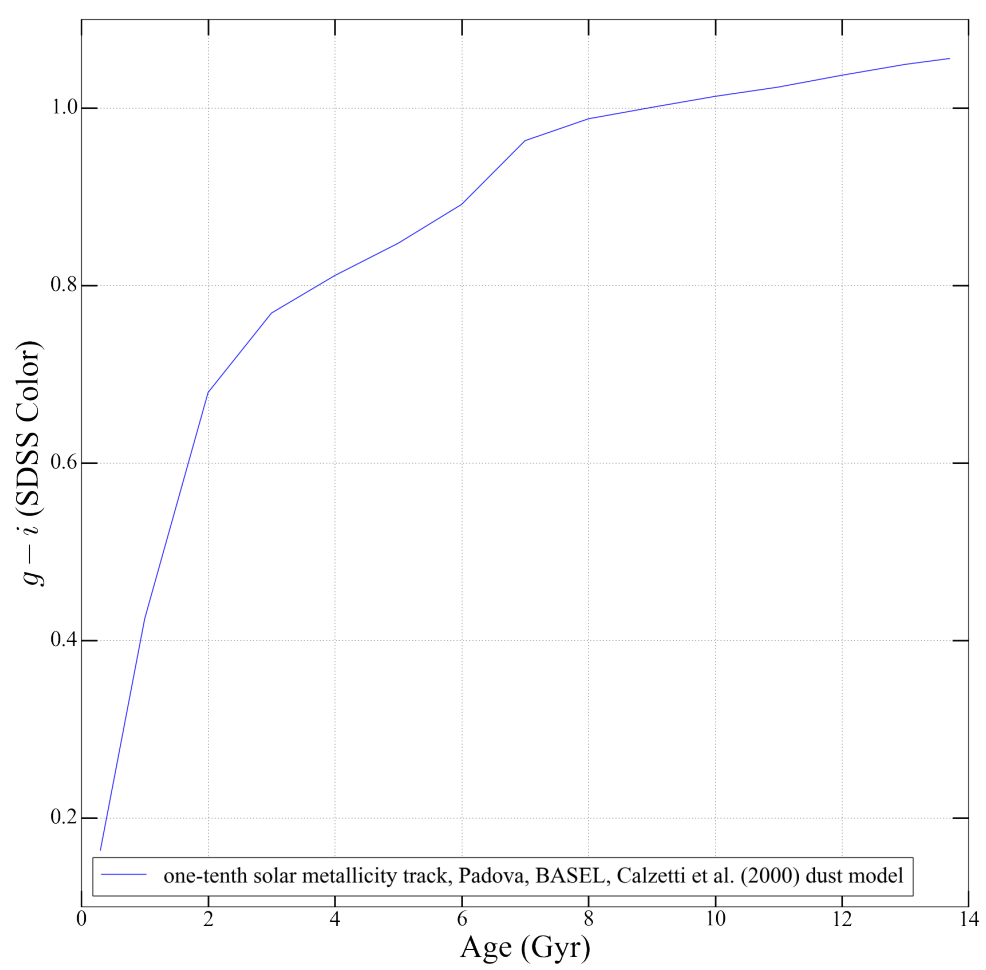

Figure 4.30: FSPS model result with one-tenth solar metallicity.

UDGs showed $g-i$ color fits between 0.4 and 0.6 , and the corresponding ages of the stellar population with one-tenth of the solar metallicity are 0.9-1.7 Gyr, as seen in Figure 4.31. This indicated an earlier formation history for the stars of UDGs based on our results. The normal galaxies with the same stellar population parameter of metallicity, and with $g-i$ colors of $0.9-1.0$ as obtained in our work, occupied a different age interval of 6.0-9.0 Gyr, as shown in Figure 4.32. 


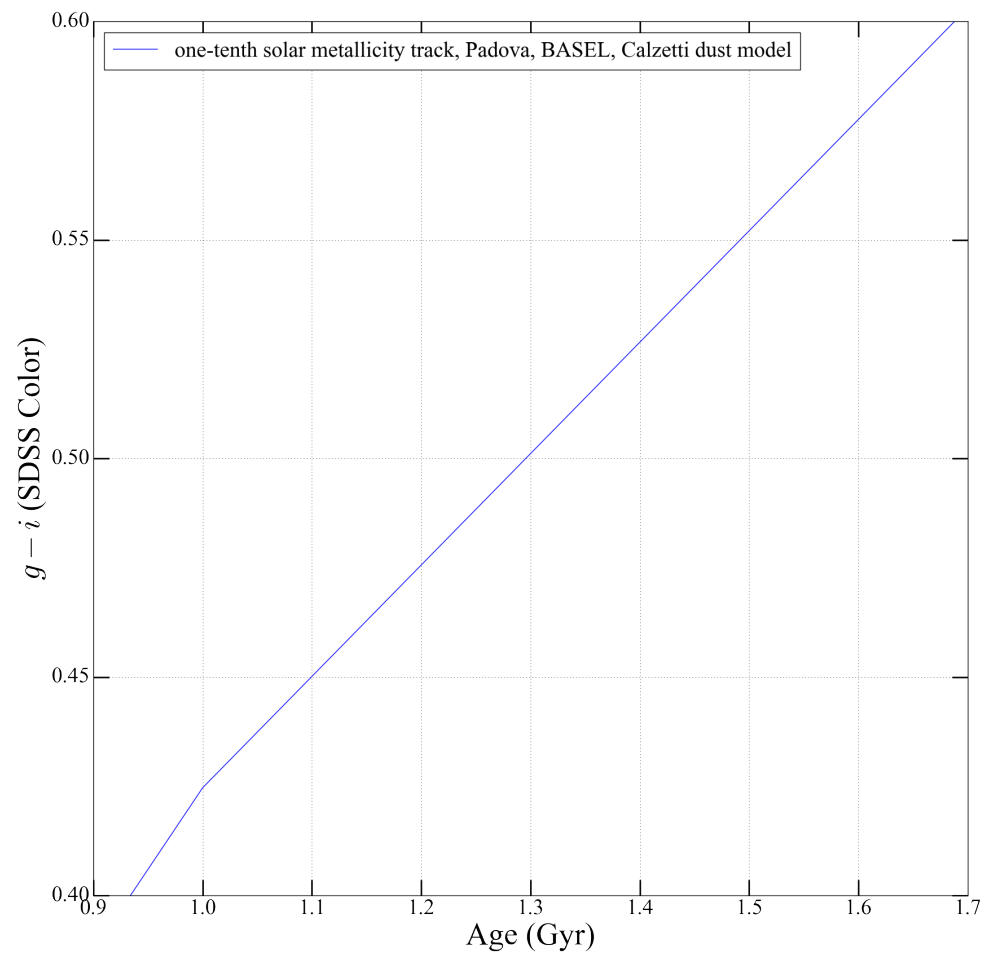

Figure 4.31: UDGs: FSPS model result with one-tenth solar metallicity. 


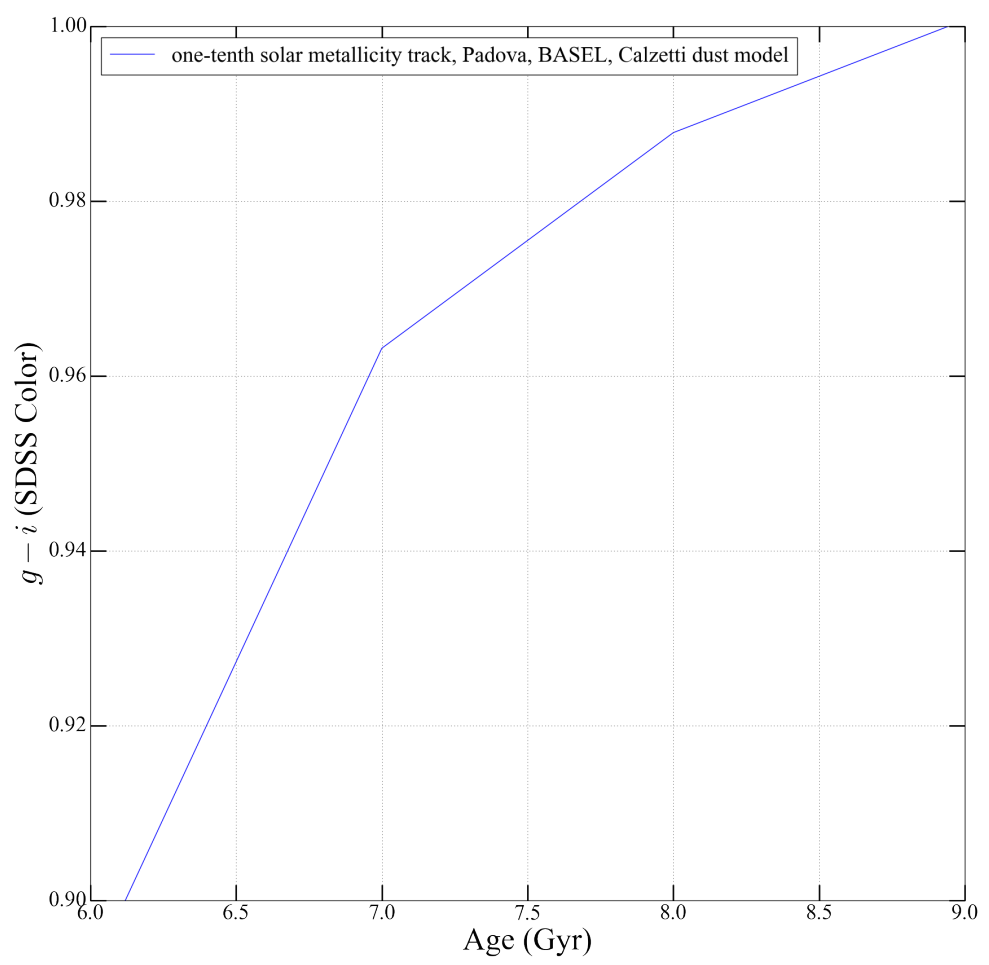

Figure 4.32: Normal galaxies: FSPS model result with one-tenth solar metallicity.

Then, we held the age constant at 10 Gyr and explored the variation in the metallicity parameter through the FSPS algorithm. Figure 4.33 shows that the resulting curve did not reach the UDG colors since according to this model and our chosen parameters, the $g-i$ colors did not go lower than about 0.8 . On the other hand, a 7 Gyr track did trace the $g-i$ interval of lower values, as shown in Figure 4.34 . 


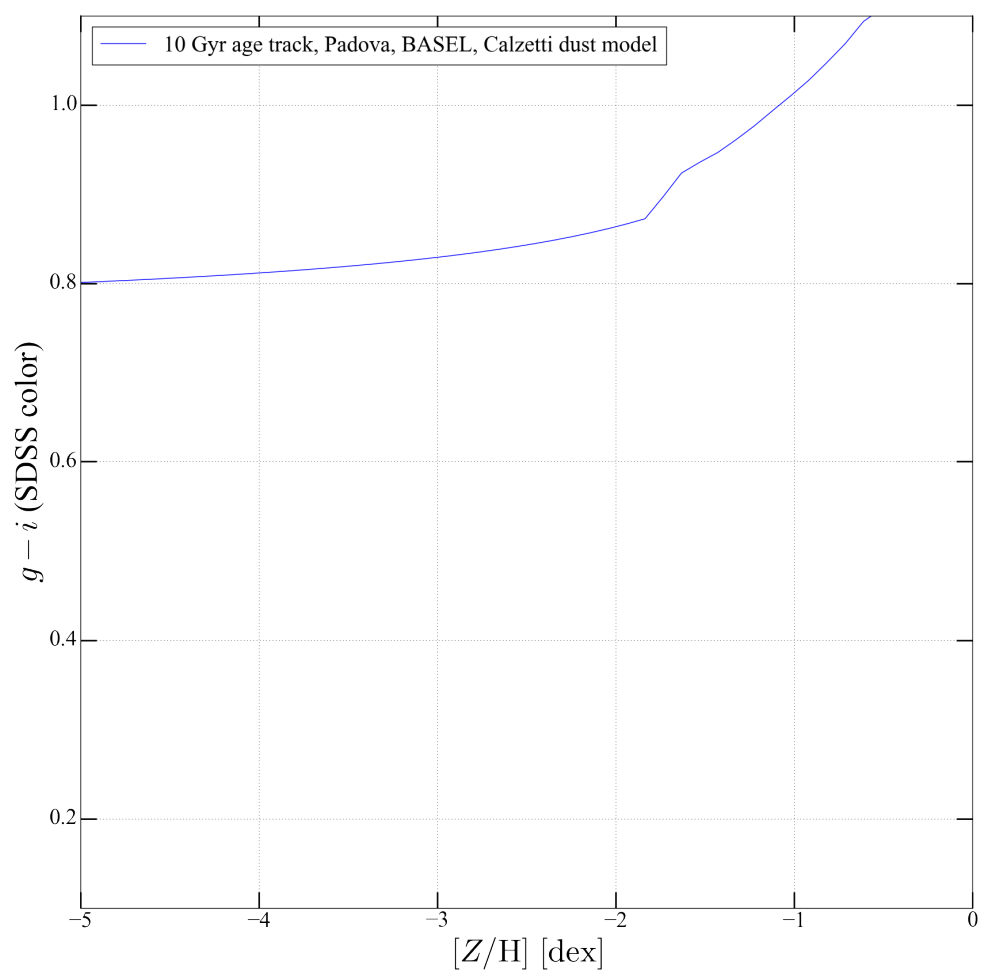

Figure 4.33: Metallicities from FSPS with a 10 Gyr track. 


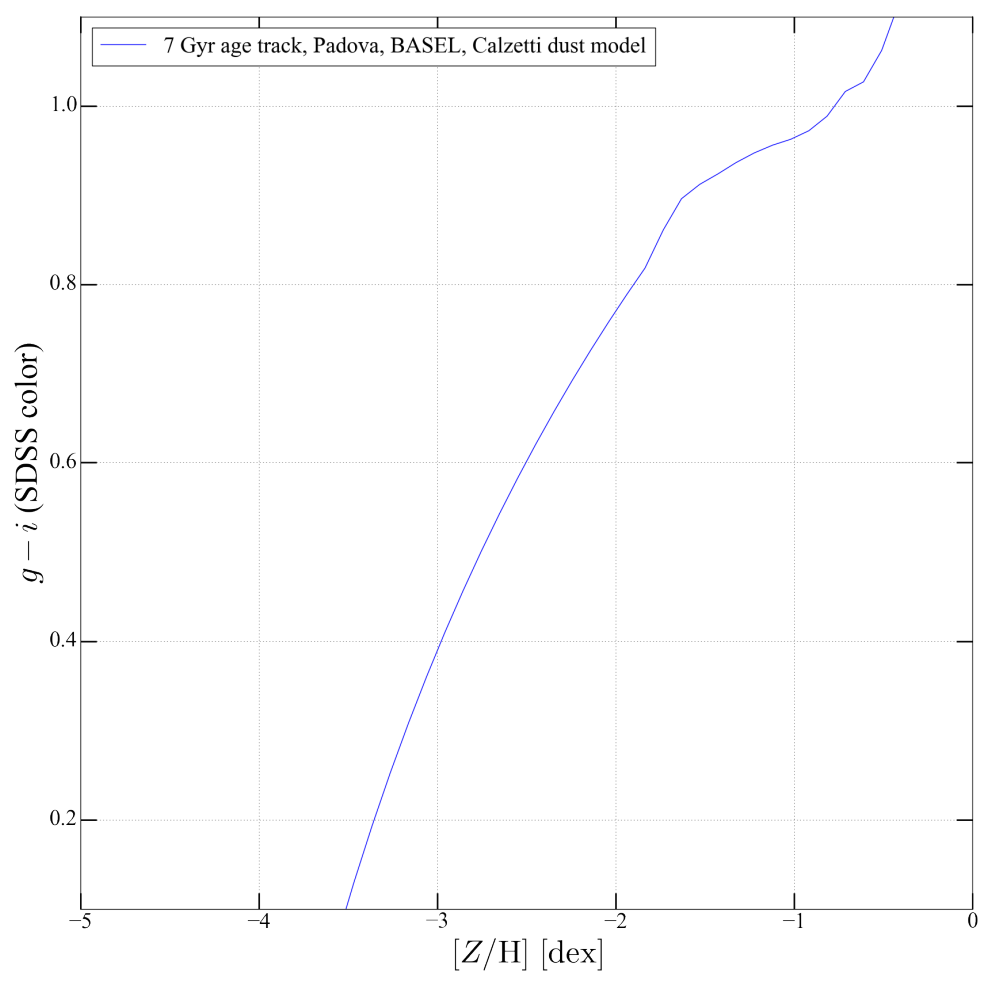

Figure 4.34: Metallicities from FSPS with a 7 Gyr track. 


\section{CHAPTER 5}

\section{SUMMARY}

\section{$5.1 \quad$ Discussion}

Here we discuss what this work has shown about the color-magnitude diagrams of UDGs. A color-magnitude (CM) diagram provides a way to visualize the broadband photometry data collected by a telescope. Analogous to the stars on the Hertzsprung-Russell diagram, galaxies do not exhibit a random distribution on the CM plot; rather they occupy distinct areas. The ultra-diffuse galaxies have been plotted on the CM diagram by Koda et al. (2015) using archival Subaru data. The authors used the Subaru $B-R$ color and the Subaru $R$ magnitude data from Yamanoi et al. (2012). These data from Yamanoi et al. (2012) were available only for 232 UDGs out of about a thousand discovered by van Dokkum et al. (2015a) and later by Koda et al. (2015). They concluded that the UDGs belonged to the red sequence of the Coma cluster of galaxies and were essentially "red and dead," i.e., they did not exhibit any active star formation. However, upon reexamination of their CM plot, several UDGs were observed to lie outside of the red sequence band and this was not explained in their paper. (Note that there was a slight difference between the published catalog data and the original paper's CM plot data, possibly because of some modifications made by the authors in the interval between the publication of the Koda et al. (2015) paper and the release of the catalog by Yagi et al. (2016).)

The Koda et al. (2015) paper did not include the catalog of the Coma cluster UDGs. It was released the following year in the paper Yagi et al. (2016) via a text-format "Subaru UDG" catalog. Dr. Romanowsky unpacked this catalog and modified it for python programming use (priv. comm.). He consequently re-created 
the CM plot with the Yagi et al. (2016) catalog data, but took it one step further; he separated the galaxies into two groups: inner and outer cluster galaxies. Inner galaxies were closer to the cluster center and populated the space within 0.5 degrees of the center of the Coma cluster. The center of the Coma cluster had the coordinates (R.A., Decl.) as (194.9531, 27.9807) in degrees from NED. Dr. Romanowsky noticed an initial visual trend in which the core and outskirts ultra-diffuse galaxies exhibited an offset in their red sequence bands (priv. comm.).

The scope of this research was the color-magnitude diagram of UDGs. This work was inspired by Dr. Romanowsky's exploratory detection of the difference between inner and outer populations in the CM plot. We continued this line of examination of the data by quantifying the observed trends.

In order to produce the CMD for normal galaxies, we used the R.A., Decl. data to identify member galaxies in the Coma cluster. Then, we used the SDSS CrossID tool to extract the SDSS ugriz magnitudes. This process was not possible for the faint ultra-diffuse galaxies.

To construct the CMD for ultra-diffuse galaxies, we used two pipelines to obtain the SDSS $g-i$ color and the SDSS $r$ magnitude. To obtain the $g-i$ color, we used the published Subaru $B-R$ colors from the Yagi et al. (2016) catalog for the 232 UDGs. Then, we converted the Subaru $B-R$ colors to the normal $B-R$ colors using equation 3.3. Next, we executed a final conversion from the normal $B-R$ colors to the SDSS $g-i$ colors using equation 4.1. For the luminosity axis, we used the SDSS $r$ magnitude which was obtained via equation 3.10. In this equation the $g$ magnitude was obtained through transformations and the $g-r$ color was obtained via a $g-r$ vs. $g-i$ color-color linear fit.

Fainter objects have larger magnitudes. Recall that magnitudes have logarithmic behavior. The CMD plot in Figure 4.26 shows that UDGs are very faint objects. 
The inner and outer population slopes for the UDGs do not run in a parallel offset to the slopes of the inner and outer populations of normal galaxies in the Coma cluster. A larger color indicates a redder galaxy. Thus, the CMD plot with UDGs shows that the inner and outer UDGs seem to represent different populations with somewhat distinct color features since the inner and outer populations of UDGs occupy different areas relative to each other on the CM diagram. The inner population of UDGs is closer to the center of the galaxy, and as expected is redder overall since they have larger $g-i$ colors. The trends in the CMD diagrams have been calculated using the basic linear regression model in the python scipy.stats package.

There are a few "bluer" UDG outliers with $0.6<g-i<0.9$ values. More work needs to be done to look individually at the outlying UDGs on the color-magnitude plot in order to investigate their properties and why they might be outliers. We also identified a list of coordinates via visual scouting of available sky surveys such as $H S T$, the HSC, and the SDSS. This preliminary list of potential targets needs to be further analyzed.

In conclusion, this work compared inner and outer populations of normal galaxies and ultra-diffuse galaxies in the Coma cluster in color-magnitude space. We used data from available astronomical catalogs, archives, databases, and surveys. The limitation of this research is the lack of photometric data and observations of UDGs.

The next step for this work is to streamline more robust transformations to reduce the amount of accumulated errors. Furthermore, the analysis of ultra-diffuse galaxies can be enriched in the future by stellar population analysis and by more spectroscopic observations. We plan to look visually for more UDGs in the astronomical archives, to investigate visually unusual UDGs on the color-magnitude 
space, and to assess whether or not UDGs have satellites. 


\section{BIBLIOGRAPHY}

Adami, C., Picat, J. P., Savine, C., et al. 2006, A\&A, 451, 1159

Albareti, F. D., Allende Prieto, C., Almeida, A., et al. 2016, ArXiv e-prints, SDSS

Collaboration, arXiv:1608.02013

Amorisco, N. C., \& Loeb, A. 2016, MNRAS, 459, L51

Beasley, M. A., Romanowsky, A. J., Pota, V., et al. 2016, ApJ, 819, L20

Bellazzini, M., Belokurov, V., Magrini, L., et al. 2017, MNRAS, 467, 3751

Bessell, M. S. 2005, ARAA, 43, 293

Binney, J., \& Merrifield, M. 1998, Galactic Astronomy

Brown, T. M., Tumlinson, J., Geha, M., et al. 2014, MemSAI, 85, 493

Bullock, J. S. 2010, ArXiv e-prints, arXiv:1009.4505

Burkert, A. 2017, ApJ, 838, 93

Cardamone, C., Schawinski, K., Sarzi, M., et al. 2009, MNRAS, 399, 1191

Conroy, C., \& Gunn, J. E. 2010, ApJ, 712, 833

Conroy, C., Gunn, J. E., \& White, M. 2009, ApJ, 699, 486

Conselice, C. J., Wilkinson, A., Duncan, K., \& Mortlock, A. 2016, ApJ, 830, 83

Consolandi, G., Gavazzi, G., Fumagalli, M., Dotti, M., \& Fossati, M. 2016, A\&A, 591, A38

Cousins, A. W. J. 1976, MmRAS, 81, 25

Da Costa, G. S. 1992, in IAU Symposium, Vol. 149, The Stellar Populations of Galaxies, ed. B. Barbuy \& A. Renzini, 191

Di Cintio, A., Brook, C. B., Dutton, A. A., et al. 2017, MNRAS, 466, L1

Ferguson, H. C., \& Binggeli, B. 1994, AApR, 6, 67

Geller, M. J., \& Huchra, J. P. 1989, Science, 246, 897

Griffith, R. L., Tsai, C.-W., Stern, D., et al. 2011, ApJ, 736, L22 
Gunn, J. E., Siegmund, W. A., Mannery, E. J., et al. 2006, AJ, 131, 2332

Hodson, A. O., \& Zhao, H. 2017, ArXiv e-prints, arXiv:1703.03463

Hubble, E. 1926, Contributions from the Mount Wilson Observatory / Carnegie Institution of Washington, 324, 1

Hubble, E. P. 1936, The realm of the nebulae, Vol. 25 (Yale University Press)

Iye, M., Karoji, H., Ando, H., et al. 2004, PASJ, 56, 381

Janssens, S., Abraham, R., Brodie, J., et al. 2017, ApJ, 839, L17

Jensen, J. B., Blakeslee, J., Cho, H., et al. 2015, in American Astronomical Society Meeting Abstracts, Vol. 225, American Astronomical Society Meeting Abstracts, 253.05

Jester, S., Schneider, D. P., Richards, G. T., et al. 2005, AJ, 130, 873

Johnson, H. L., \& Morgan, W. W. 1953, ApJ, 117, 313

Kadowaki, J., Zaritsky, D., \& Donnerstein, R. L. 2017, ApJ, 838, L21

Koda, J., Yagi, M., Yamanoi, H., \& Komiyama, Y. 2015, ApJ, 807, L2

Landolt, A. U. 1983, AJ, 88, 439

Leisman, L., Haynes, M. P., Giovanelli, R., \& ALFALFA Almost Darks Team. 2017, in American Astronomical Society Meeting Abstracts, Vol. 229, American

Astronomical Society Meeting Abstracts, 132.06

Martínez-Delgado, D., Läsker, R., Sharina, M., et al. 2016, AJ, 151, 96

Mei, S., Blakeslee, J. P., Côté, P., et al. 2007, ApJ, 655, 144

Mieske, S., Infante, L., Hilker, M., et al. 2005, A\&A, 430, L25

Mihos, J. C., Durrell, P. R., Ferrarese, L., et al. 2015, ApJ, 809, L21

Miyazaki, S., Komiyama, Y., Sekiguchi, M., et al. 2002, PASJ, 54, 833

Mo, H., van den Bosch, F. C., \& White, S. 2010, Galaxy Formation and Evolution

Okabe, N., Futamase, T., Kajisawa, M., \& Kuroshima, R. 2014, ApJ, 784, 90

Peng, E. W., \& Lim, S. 2016, ApJ, 822, L31

Phillipps, S., Drinkwater, M. J., Gregg, M. D., \& Jones, J. B. 2001, ApJ, 560, 201 
Reaves, G. 1956, AJ, 61, 69

Rees, M. J., \& Volonteri, M. 2007, in IAU Symposium, Vol. 238, Black Holes from Stars to Galaxies - Across the Range of Masses, ed. V. Karas \& G. Matt, 51-58

Román, J., \& Trujillo, I. 2017, MNRAS, 468, 703

Tinsley, B. M. 1968, ApJ, 151, 547

—. 1980, Fund. Cosmic Phys., 5, 287

van den Bergh, S. 1979, JRASC, 73, 198

van der Burg, R. F. J., Muzzin, A., \& Hoekstra, H. 2016, A\&A, 590, A20

van Dokkum, P., Abraham, R., Brodie, J., et al. 2016, ApJ, 828, L6

van Dokkum, P. G., Abraham, R., Merritt, A., et al. 2015a, ApJ, 798, L45

van Dokkum, P. G., Romanowsky, A. J., Abraham, R., et al. 2015b, ApJ, 804, L26

Watson, D. F., Berlind, A. A., McBride, C. K., Hogg, D. W., \& Jiang, T. 2012, ApJ, 749, 83

Yagi, M., Koda, J., Komiyama, Y., \& Yamanoi, H. 2016, ApJS, 225, 11

Yamanoi, H., Komiyama, Y., Yagi, M., et al. 2012, AJ, 144, 40

York, D. G., Adelman, J., Anderson, Jr., J. E., et al. 2000, AJ, 120, 1579

Yozin, C., \& Bekki, K. 2015, MNRAS, 452, 937 